\title{
The effect of pharmacotherapy on prostate volume, prostate perfusion and prostate-specific antigen (prostate morphometric parameters) in patients with lower urinary tract symptoms and benign prostatic obstruction. A systematic review and meta-analysis
}

\author{
Vasileios Sakalis ${ }^{1,3}$, Anastasia Gkotsi ${ }^{1}$, Dimitra Charpidou ${ }^{1}$, Petros Tsafrakidis² ${ }^{2}$ Apostolos Apostolidis ${ }^{3}$ \\ ${ }^{1}$ Department of Urology, Agios Pavlos General Hospital of Thessaloniki, Thessaloniki, Greece \\ ${ }^{2}$ Department of Urology, Fife NHS Trust, Fife, United Kingdom \\ ${ }^{3} 2^{\text {nd }}$ Department of Urology, Aristotle University of Thessaloniki, Thessaloniki, Greece \\ Citation: Sakalis V, Gkotsi A, Charpidou D, Tsafrakidis P, Apostolidis A. The effect of pharmacotherapy on prostate volume, prostate perfusion and prostate-specific \\ antigen (prostate morphometric parameters) in patients with lower urinary tract symptoms and benign prostatic obstruction. A systematic review and meta- \\ analysis. Cent European J Urol. 2021; 74: 388-421.
}

Article history

Submitted: Nov. 16, 2020 Accepted: June 30, 2021

Published online: Aug. 11, 2021

Corresponding author Vasileios Sakalis Agios Pavlos General Hospital of Thessaloniki 161 Ethnikis Antistaseos 55132 Thessaloniki, Greece

phone: +30 6987402020 vsakkalis@hotmail.com
Introduction The clinical effect of pharmacotherapy on prostate morphometric parameters is largely unknown. The sole exception is $5 \alpha$-reductase inhibitors (5-ARI) that reduce prostate volume and prostate-specific antigen (PSA). This review assesses the effect of pharmacotherapy on prostate parameters effect on prostate parameters, namely total prostate volume (TPV), transitional zone volume (TZV), PSA and prostate perfusion.

Material and methods We conducted a systematic review and meta-analysis of randomized controlled trials (RCTs) reporting on morphometric parameters' changes after pharmacotherapy, as primary or secondary outcomes. The study followed the Preferred Reporting Items for Systematic Reviews and Meta-Analyses (PRISMA) statement. RCTs' quality was assessed by the Cochrane tool and the criteria of the Agency for Healthcare Research and Quality. The effect magnitude was expressed as standard mean difference (SMD). The study protocol was published on PROSPERO (CRD42020170172).

Results Sixty-seven RCTs were included in the review and 18 in the meta-analysis. The changes after alpha-blockers are comparable to placebo. Long-term studies reporting significant changes from baseline, result from physiologic growth. Finasteride and dutasteride demonstrated large effect sizes in TPV reduction ([SMD]:-1.15 (95\% Cl:-1.26 to-1.04, $\mathrm{p}<0.001$, and [SMD]:-0.66 (95\% Cl:-0.83 to- $0.49, \mathrm{p}<0.001$, respectively), and similar PSA reductions. Dutasteride's effect appears earlier ( $1^{\text {st }} v s$ s $3^{\text {rd }}$ month), the changes reach a maximum at month 12 and are sustained thereafter. Phosphodiesterase-5 (PDE-5) inhibitors have no effect on morphometric parameters. Phytotherapy's effect on TPV is non-significant [SMD]: 0.12 (95\% Cl:-0.03 to 0.27, p = 0.13). Atorvastatin reduces TPV as compared to placebo $(-11.7 \%$ vs $+2.5 \%, p<0.01)$. Co-administration of testosterone with dutasteride spares the prostate from the androgenic stimulation as both TPV and PSA are reduced significantly.

Conclusions The 5-ARIs show large effect size in reducing TPV and PSA. Tamsulosin improves perfusion but no other effect is evident. PDE-5 inhibitors and phytotherapy do not affect morphometric parameters. Atorvastatin reduces TPV and PSA as opposed to testosterone supplementation.

\section{Key Words: prostate volume changes 〈' prostate perfusion 〈〉 lower urinary tract pharmacotherapy () morphometric parameters}




\section{INTRODUCTION}

Benign prostatic obstruction (BPO) is a common cause of lower urinary tract symptoms (LUTS) in men older than 50 years [1]. Benign prostatic enlargement (BPE) is defined as prostatic enlargement due to histologic benign prostatic hyperplasia [2]. $\mathrm{BPO}$ involves the static component or the physical mass of the prostate and the dynamic component or smooth muscle tone of the prostate stroma and the bladder neck $[1,2]$. It is reasonable to assume a potential relation between prostate size, degree of obstruction and LUTS severity, but populationbased studies failed to demonstrate a direct link [3]. Prostate morphometric parameters are prognostic indicators of BPE progression. Data analysis from the placebo arm of Medical Therapy of Prostatic Symptoms (MTOPS) trial showed that men with baseline total prostate volume (TPV) $31 \mathrm{ml}$ and prostate-specific antigen (PSA) of $1.6 \mathrm{ng} / \mathrm{dl}$ or greater are at significantly higher risk of BPE progression, defined as a 4-point or more increase in AUA-SS, acute urinary retention, urinary incontinence, renal insufficiency or recurrent urinary tract infections [4]. Baseline flow rate, post-void residual and age were the additional predictors. TPV and PSA are among the baseline factors which could predict conservative treatment failure and/or the need for combination therapy [5]. Baseline PSA is higher in men with larger prostates and is associated with higher annual volume increase $(2.2 \%)$ compared to smaller prostates (1.7\%) [6]. However, a multivariate analysis of the Baltimore Longitudinal Study of Aging in 242 men without prostate cancer, reported no correlation between PSA or PSA changes and annual prostate growth rate during 4.2 years of follow-up [6]. The median rate of TPV and PSA change per year was $0.6 \mathrm{ml}$ and $0.03 \mathrm{ng} / \mathrm{ml}$ respectively.

Existing data supports the hypothesis that ischemia of the lower urinary tract may cause BPE and LUTS. Azadzoi et al. were first to document bladder dysfunction and increased prostate contractility in an animal model of pelvic atherosclerosis [7]. The underlying mechanism of ischemic injury involves oxidative stress, free radical injury to smooth muscle cells, epithelium, mitochondria, endoplasmic reticulum and nerve fibers, impairment of the nitric oxide (NO/cGMP) pathway, activation of degenerative processes and deposition of collagen [7]. Chronic ischemia induces prostate stromal fibrosis, decreases cGMP and increases prostate tissue sensitivity to contractile stimuli [7].

The clinical effect of pharmacotherapy on prostate morphometric parameters is largely unknown. The sole exception is $5 \alpha$-reductase inhibitors (5-ARI) which reduce TPV, transitional zone volume (TZV) and PSA. There is preclinical evidence that all medications influence prostate volume or perfusion. Experiments have shown the anti-apoptotic effect of sympathomimetics, and the potent apoptotic effect on human prostate cancer cell cultures of quinazoline-based $\alpha$-blockers [8].

Phosphodiesterase-5 (PDE-5) inhibitors influence prostate cell proliferation via upregulation of $\mathrm{NO} / \mathrm{cGMP}$ and Rho-kinase activity $[9,10]$. Evidence supports that finasteride reduces prostate blood flow via downregulation of vascular endothelial growth factor (VEGF) [11]. Tamsulosin antagonizes vesical arteries adrenoceptors, thus improving LUT perfusion [12]. PDE5 inhibitors improve perfusion via the reduction of endothelin-1 levels and regulation of vascular smooth muscle cells proliferation [10].

This review aims to investigate the effect of both urological and non-urological medication on prostate morphometric parameters, namely TPV, TZV, PSA and prostate perfusion.

\section{MATERIAL AND METHODS}

\section{Literature search}

This systematic review was performed according to the Preferred Reporting Items for Systematic Reviews and Meta-Analyses (PRISMA) Statement [13]. The Embase, MEDLINE, Cochrane Database of Systematic Reviews, Cochrane Central (Cochrane Health Technology Assessment, Database of Abstracts of Reviews of Effects, Health Economics Evaluations Database) and Google Scholar were searched with no restriction on publication date. Additional sources for articles were the reference lists of included studies and relevant review articles.

\section{Study selection}

We included randomized-controlled trials (RCTs) of adult men with LUTS due to BPE, who received pharmacotherapy, and reported post-intervention changes of prostate parameters as primary or secondary outcome. The included studies had 10 participants minimum, were written in English language and used ultrasound or MRI to assess morphometric parameters. There was no restriction in study duration. In the event of open extension of double-blind studies, only data from the double-blind period were included. If data were not reported separately, studies were excluded.

Two reviewers (AG and DC) screened the titles and abstracts of identified records, and the full text of potentially eligible records was evaluated using a stan- 
dardized form. Disagreement was resolved by discussion. If there was no agreement, a third independent party acted as an arbitrator (VS).

\section{Data extraction}

Data from eligible studies were extracted in duplicate. Discrepancies were resolved by a third reviewer. The variables assessed included the year of publication, number of randomized subjects, number of subjects who completed the follow up, baseline values and post treatment changes in morphometric parameters presented as mean ( \pm standard deviation) and percentage changes from baseline.

\section{Risk of bias and study quality assessment}

Risk of bias (RoB) was assessed using the revised version of Cochrane Collaboration's RoB Assessment tool [14]. Two reviewers (AG and DC) independently assessed RoB in each study, while a third reviewer (VS) acted as an arbitrator. The RoB was considered high if the confounder had not been considered by the individual study. The RoB tables were developed in Review Manager 5.3 (RevMan-Informatics and Knowledge Management Department, Cochrane, London, UK).

To ensure reliability and validity of measures and reported measurements, each included RCT had an overall rating based on the criteria developed by Agency for Healthcare Research and Quality (AHRQ). The ratings were 'Low-risk', 'Moderate-risk' or 'Highrisk' $[15,16]$. The RCTs should have been characterized as low risk in measurement bias (points $3 \mathrm{~d} \& 3 \mathrm{e}$ ) based on the criteria developed by AHRQ.

\section{Statistical analysis}

The primary outcome was the post-intervention changes in TPV. The secondary outcomes were the changes in TZV, PSA and prostate perfusion as defined by the trialist. Owing to the expected heterogeneity, a narrative synthesis of all included studies was planned [17]. Data are presented as post-treatment absolute mean changes $( \pm \mathrm{SD})$ and percentage changes.

Statistical heterogeneity was tested using chi-square test. A value of $\mathrm{p}<0.10$ or I2 $>50 \%$ was used to define heterogeneity. A list of potential confounders was developed a priori: use of LUTS-related medications, follow-up duration, LUTS not related to BPE, previous catheter use, previous LUT surgery and history of LUT malignancy.

A meta-analysis was considered for each endpoint if two or more RCTs had similar study design, dos- ing scheme and follow-up duration. Meta-analysis was conducted using RevMan. The effect magnitude was expressed as standard mean difference (SMD) with $95 \%$ confidence interval (CI) for continuous outcomes. The treatment effect size was considered small for SMD values of 0-0.2, moderate for SMD range $0.2-0.8$ and large if SMD was $>0.8$.

\section{RESULTS}

\section{Evidence acquisition}

\section{Study selection}

Sixty-seven RCTs were eligible for inclusion (Figure 1). Eighteen were eligible for quantitative synthesis. The search was updated in October 2020.

\section{Study characteristics}

We identified 28 placebo-controlled RCTs and 39 nonplacebo RCTs. Since the included RCTs had 2 or more study arms, we studied 36 active medications versus placebo comparisons and 48 active medications versus active medication comparisons. Phytotherapy's effect on morphometric parameters was assessed in 18 comparisons, $\alpha$-blockers' effect in 18 comparisons, 5-ARI's effect in 23 comparisons, PDE5's effect in 6 comparisons, combination treatments

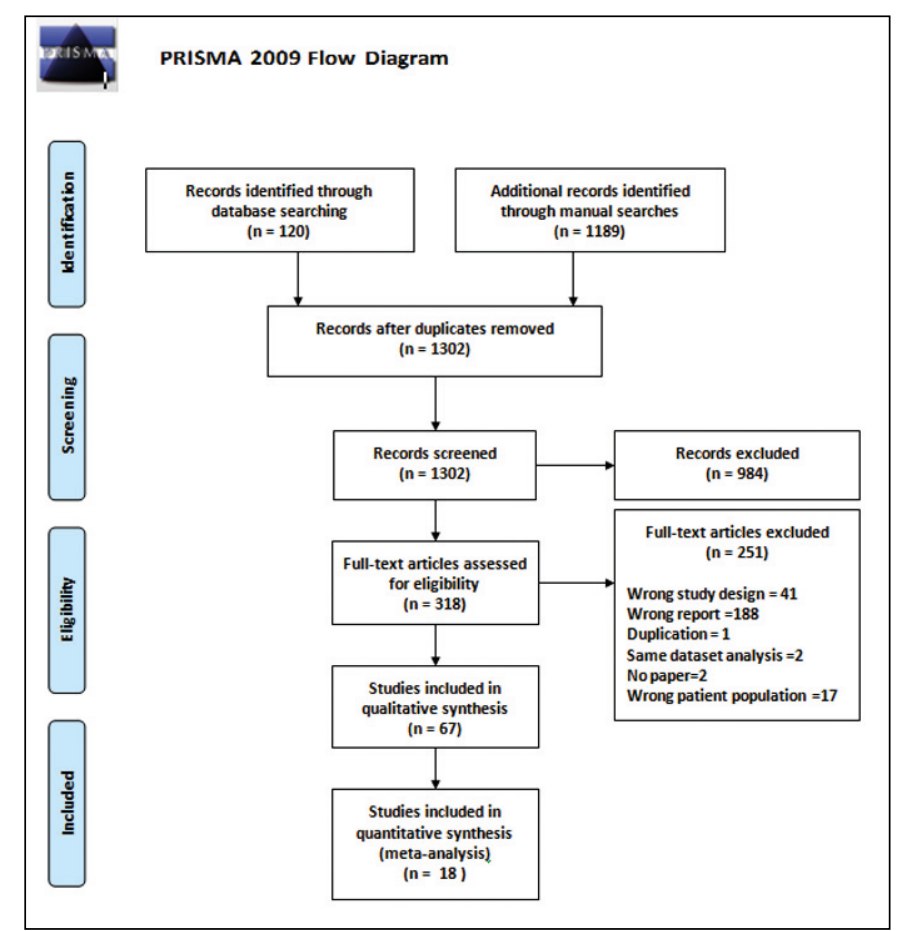

Figure 1. Preferred Reporting Items for Systematic Reviews and Meta-Analyses (PRISMA) flow chart. 
Table 1. The characteristics of included trials

\begin{tabular}{|c|c|c|c|c|c|c|c|c|c|}
\hline $\begin{array}{c}\text { Study, } \\
\text { [reference] }\end{array}$ & $\begin{array}{l}\text { Comparator } 1, \\
\text { Daily dosage }\end{array}$ & $\begin{array}{l}\text { Comparator 2, } \\
\text { Daily dosage }\end{array}$ & $\begin{array}{c}\text { Comparator } 3, \\
\text { Daily dosage }\end{array}$ & $\begin{array}{l}\text { Comparator } 4 \text {, } \\
\text { Daily dosage }\end{array}$ & $\begin{array}{l}\text { No. subjects } \\
\text { randomized }\end{array}$ & $\begin{array}{l}\text { Duration of } \\
\text { Follow up }\end{array}$ & $\begin{array}{c}\text { Reported } \\
\text { parameters }\end{array}$ & $\begin{array}{l}\text { Primary } \\
\text { or Secondary } \\
\text { endpoints }\end{array}$ & $\begin{array}{l}\text { Study rating } \\
\text { based on } \\
\text { AHRQ criteria }\end{array}$ \\
\hline $\begin{array}{l}\text { Lepor 1996, } \\
{[18]}\end{array}$ & $\begin{array}{l}\text { Terazosin, } \\
10 \text { mg OD }\end{array}$ & $\begin{array}{l}\text { Finasteride, } \\
5 \mathrm{mg} \mathrm{OD}\end{array}$ & $\begin{array}{l}\text { Terazosin } \\
10 \text { mg OD plus } \\
\text { Finasteride, } \\
5 \text { mg OD }\end{array}$ & Placebo & 1230 & 12 months & TPV, PSA & Secondary & Low Risk \\
\hline $\begin{array}{l}\text { McConnell } \\
\text { 2003, [19] }\end{array}$ & $\begin{array}{l}\text { Doxazosin, } \\
4 \text { or } 8 \text { mg OD }\end{array}$ & $\begin{array}{l}\text { Finasteride, } \\
5 \mathrm{mg} \mathrm{OD}\end{array}$ & $\begin{array}{c}\text { Doxazosin, } 4 \\
\text { or } 8 \text { mg OD plus } \\
\text { Finasteride, } \\
5 \text { mg OD }\end{array}$ & Placebo & 3047 & 4.5 years & TPV, PSA & Secondary & Low Risk \\
\hline $\begin{array}{l}\text { Yokoyama } \\
\text { 2012, [20] }\end{array}$ & $\begin{array}{c}\text { Tadalafil. } \\
2.5 \text { mg OD }\end{array}$ & $\begin{array}{l}\text { Tadalafil } \\
5 \mathrm{mg} \text { OD }\end{array}$ & $\begin{array}{l}\text { Tamsulosin, } \\
0.2 \mathrm{mg} \mathrm{OD}\end{array}$ & Placebo & 612 & 3 months & PSA & Secondary & Low Risk \\
\hline $\begin{array}{l}\text { Roehrborn } \\
2006,[21]\end{array}$ & $\begin{array}{l}\text { Alfuzosin, } \\
10 \text { mg OD }\end{array}$ & Placebo & $\mathrm{n} / \mathrm{a}$ & $\mathrm{n} / \mathrm{a}$ & 1522 & 24 months & PSA & Secondary & Low Risk \\
\hline $\begin{array}{l}\text { Roehrborn } \\
2006,[22]\end{array}$ & $\begin{array}{l}\text { Alfuzosin, } \\
10 \text { mg OD }\end{array}$ & Placebo & $\mathrm{n} / \mathrm{a}$ & $\mathrm{n} / \mathrm{a}$ & 528 & 3 months & TPV, TZV & Primary & $\begin{array}{c}\text { Moderate } \\
\text { Risk }\end{array}$ \\
\hline $\begin{array}{l}\text { Turkeri 2001, } \\
\text { [23] }\end{array}$ & $\begin{array}{l}\text { Doxazosin, } \\
4 \text { mg OD }\end{array}$ & Placebo & $\mathrm{n} / \mathrm{a}$ & $\mathrm{n} / \mathrm{a}$ & 29 & 4 weeks & TPV, PSA & Secondary & High Risk \\
\hline $\begin{array}{l}\text { Debruyne } \\
\text { 2002, [24] }\end{array}$ & $\begin{array}{l}\text { Tamsulosin, } \\
0.4 \text { mg OD }\end{array}$ & $\begin{array}{l}\text { Serenoa repens, } \\
320 \text { mg OD }\end{array}$ & $\mathrm{n} / \mathrm{a}$ & $\mathrm{n} / \mathrm{a}$ & 704 & 12 months & TPV, PSA & Primary & Low Risk \\
\hline $\begin{array}{l}\text { Sengupta } \\
\text { 2011, [25] }\end{array}$ & $\begin{array}{l}\text { Tamsulosin, } \\
0.4 \text { mg OD }\end{array}$ & $\begin{array}{l}\text { Phytotherapy } \\
\text { (Non-Sr), OD }\end{array}$ & $\mathrm{n} / \mathrm{a}$ & $\mathrm{n} / \mathrm{a}$ & 46 & 3 months & TPV & Secondary & $\begin{array}{c}\text { Moderate } \\
\text { Risk }\end{array}$ \\
\hline $\begin{array}{l}\text { Latil 2015, } \\
{[26]}\end{array}$ & $\begin{array}{l}\text { Tamsulosin, } \\
0.4 \text { mg OD }\end{array}$ & $\begin{array}{l}\text { Hexanic Extract } \\
\text { Serenoa repens, } \\
320 \text { mg OD }\end{array}$ & $\mathrm{n} / \mathrm{a}$ & $\mathrm{n} / \mathrm{a}$ & 203 & 3 months & TPV & Secondary & High Risk \\
\hline $\begin{array}{l}\text { Pande 2014, } \\
{[27]}\end{array}$ & $\begin{array}{l}\text { Tamsulosin, } \\
0.4 \text { mg OD }\end{array}$ & $\begin{array}{l}\text { Silodosin, } \\
8 \mathrm{mg} \mathrm{OD}\end{array}$ & $\mathrm{n} / \mathrm{a}$ & $\mathrm{n} / \mathrm{a}$ & 61 & 3 months & TPV & Secondary & $\begin{array}{c}\text { Moderate } \\
\text { Risk }\end{array}$ \\
\hline $\begin{array}{l}\text { Karami 2016, } \\
{[28]}\end{array}$ & $\begin{array}{l}\text { Tamsulosin, } \\
0.4 \text { mg OD }\end{array}$ & $\begin{array}{l}\text { Tadalafil, } \\
20 \text { mg OD }\end{array}$ & $\mathrm{n} / \mathrm{a}$ & $\mathrm{n} / \mathrm{a}$ & 119 & 3 months & PSA & Primary & High Risk \\
\hline $\begin{array}{l}\text { Griwan } \\
\text { 2014, [99] }\end{array}$ & $\begin{array}{l}\text { Tamsulosin, } \\
0.4 \text { mg OD }\end{array}$ & $\begin{array}{l}\text { Naftopidil, } \\
75 \text { mg OD }\end{array}$ & $\mathrm{n} / \mathrm{a}$ & $\mathrm{n} / \mathrm{a}$ & 60 & 3 months & TPV & Secondary & $\begin{array}{c}\text { Moderate } \\
\text { Risk }\end{array}$ \\
\hline $\begin{array}{l}\text { HIzli 2007, } \\
\text { [29] }\end{array}$ & $\begin{array}{l}\text { Tamsulosin, } \\
0.4 \text { mg OD }\end{array}$ & $\begin{array}{l}\text { Serenoa repens, } \\
320 \text { mg OD }\end{array}$ & $\mathrm{n} / \mathrm{a}$ & $\mathrm{n} / \mathrm{a}$ & 40 & 6 months & TPV, PSA & Secondary & High Risk \\
\hline $\begin{array}{l}\text { Odysanya } \\
\text { 2017, [30] }\end{array}$ & $\begin{array}{l}\text { Tamsulosin, } \\
0.4 \text { mg OD }\end{array}$ & $\begin{array}{c}\text { Finasteride, } \\
5 \mathrm{mg} \text { OD }\end{array}$ & $\begin{array}{l}\text { Tamsulosin, } \\
0.4 \text { mg OD plus } \\
\text { Finasteride, } \\
5 \text { mg OD }\end{array}$ & $\mathrm{n} / \mathrm{a}$ & 60 & 6 months & TPV & Secondary & High Risk \\
\hline $\begin{array}{l}\text { Morgia } \\
2014,[31]\end{array}$ & $\begin{array}{l}\text { Tamsulosin, } \\
0.4 \text { mg OD }\end{array}$ & $\begin{array}{l}\text { Phytotherapy } \\
\text { (Non-Sr) }\end{array}$ & $\begin{array}{l}\text { Tamsulosin, } \\
0.4 \text { mg OD plus } \\
\text { Phytotherapy } \\
\text { (Non-Sr) }\end{array}$ & $\mathrm{n} / \mathrm{a}$ & 150 & 12 months & TPV, PSA & Secondary & Low Risk \\
\hline $\begin{array}{l}\text { Roehrborn } \\
\text { 2010, [32] }\end{array}$ & $\begin{array}{l}\text { Tamsulosin, } \\
0.4 \text { mg OD }\end{array}$ & $\begin{array}{l}\text { Dutasteride, } \\
0.5 \mathrm{mg} \mathrm{OD}\end{array}$ & $\begin{array}{l}\text { Tamsulosin, } \\
0.4 \text { mg OD plus } \\
\text { Dutasteride, } \\
0.5 \text { mg OD }\end{array}$ & $\mathrm{n} / \mathrm{a}$ & 3221 & 4 years & TPV, PSA & Secondary & Low Risk \\
\hline $\begin{array}{l}\text { Debruyne } \\
\text { 1998, [33] }\end{array}$ & $\begin{array}{l}\text { Alfuzosin SR, } \\
\text { OD }\end{array}$ & $\begin{array}{l}\text { Finasteride, } \\
5 \mathrm{mg} \text { OD }\end{array}$ & $\begin{array}{l}\text { Alfuzosin SR, } \\
\text { OD plus } \\
\text { Finasteride, } \\
5 \text { mg OD }\end{array}$ & $\mathrm{n} / \mathrm{a}$ & 707 & 6 months & TPV, PSA & Secondary & Low Risk \\
\hline $\begin{array}{l}\text { Sakalis 2018, } \\
\text { [34] }\end{array}$ & $\begin{array}{l}\text { Tamsulosin, } \\
0.4 \text { mg OD }\end{array}$ & $\begin{array}{c}\text { Solifenacin, } \\
5 \text { or } 10 \text { mg OD }\end{array}$ & $\mathrm{n} / \mathrm{a}$ & $\mathrm{n} / \mathrm{a}$ & 69 & 6 months & $\begin{array}{c}\text { TPV, TZV, } \\
\text { PSA, } \\
\text { Perfusion } \\
\text { parameters }\end{array}$ & Primary & $\begin{array}{l}\text { Moderate } \\
\text { Risk }\end{array}$ \\
\hline $\begin{array}{l}\text { Andersen } \\
\text { 1995, [35] }\end{array}$ & $\begin{array}{l}\text { Finasteride, } \\
5 \mathrm{mg} \mathrm{OD}\end{array}$ & Placebo & $\mathrm{n} / \mathrm{a}$ & $\mathrm{n} / \mathrm{a}$ & 707 & 24 months & TPV, PSA & Secondary & $\begin{array}{l}\text { Moderate } \\
\text { Risk }\end{array}$ \\
\hline $\begin{array}{l}\text { Nickel 1996, } \\
{[36]}\end{array}$ & $\begin{array}{l}\text { Finasteride, } \\
5 \mathrm{mg} \mathrm{OD}\end{array}$ & Placebo & $\mathrm{n} / \mathrm{a}$ & $\mathrm{n} / \mathrm{a}$ & 613 & 24 months & TPV, PSA & Primary & Low Risk \\
\hline $\begin{array}{l}\text { McConnell } \\
1998,[37]\end{array}$ & $\begin{array}{l}\text { Finasteride, } \\
5 \mathrm{mg} \mathrm{OD}\end{array}$ & Placebo & $\mathrm{n} / \mathrm{a}$ & $\mathrm{n} / \mathrm{a}$ & 312 & 48 months & TPV & Secondary & Low Risk \\
\hline
\end{tabular}


Table 1. Continued

\begin{tabular}{|c|c|c|c|c|c|c|c|c|c|}
\hline $\begin{array}{c}\text { Study, } \\
\text { [reference] }\end{array}$ & $\begin{array}{l}\text { Comparator } 1, \\
\text { Daily dosage }\end{array}$ & $\begin{array}{l}\text { Comparator 2, } \\
\text { Daily dosage }\end{array}$ & $\begin{array}{c}\text { Comparator } 3, \\
\text { Daily dosage }\end{array}$ & $\begin{array}{l}\text { Comparator } 4 \text {, } \\
\text { Daily dosage }\end{array}$ & $\begin{array}{l}\text { No. subjects } \\
\text { randomized }\end{array}$ & $\begin{array}{l}\text { Duration of } \\
\text { Follow up }\end{array}$ & $\begin{array}{c}\text { Reported } \\
\text { parameters }\end{array}$ & $\begin{array}{l}\text { Primary } \\
\text { or Secondary } \\
\text { endpoints }\end{array}$ & $\begin{array}{l}\text { Study rating } \\
\text { based on } \\
\text { AHRQ criteria }\end{array}$ \\
\hline $\begin{array}{l}\text { Marberger } \\
1998,[38]\end{array}$ & $\begin{array}{c}\text { Finasteride, } \\
5 \mathrm{mg} \mathrm{OD}\end{array}$ & Placebo & $\mathrm{n} / \mathrm{a}$ & $\mathrm{n} / \mathrm{a}$ & 2902 & 24 months & TPV & Secondary & $\begin{array}{c}\text { Moderate } \\
\text { Risk }\end{array}$ \\
\hline $\begin{array}{l}\text { Kirby 1992, } \\
\text { [39] }\end{array}$ & $\begin{array}{c}\text { Finasteride, } \\
5 \mathrm{mg} \mathrm{OD}\end{array}$ & $\begin{array}{c}\text { Finasteride, } \\
10 \text { mg OD }\end{array}$ & Placebo & $\mathrm{n} / \mathrm{a}$ & 66 & 3 months & TPV, PSA & Secondary & High Risk \\
\hline $\begin{array}{l}\text { Finasteride } \\
\text { group 1993, } \\
\text { [40] }\end{array}$ & $\begin{array}{l}\text { Finasteride, } \\
1 \mathrm{mg} \text { OD }\end{array}$ & $\begin{array}{l}\text { Finasteride, } \\
5 \mathrm{mg} \mathrm{OD}\end{array}$ & Placebo & $\mathrm{n} / \mathrm{a}$ & 750 & 12 months & TPV, PSA & Secondary & $\begin{array}{l}\text { Moderate } \\
\text { Risk }\end{array}$ \\
\hline $\begin{array}{l}\text { Tammela } \\
\text { 1995, [41] }\end{array}$ & $\begin{array}{c}\text { Finasteride, } \\
5 \mathrm{mg} \mathrm{OD}\end{array}$ & Placebo & $\mathrm{n} / \mathrm{a}$ & $\mathrm{n} / \mathrm{a}$ & 36 & 6 months & TPV & Secondary & High Risk \\
\hline $\begin{array}{l}\text { Pannek } \\
\text { 1998, [42] }\end{array}$ & $\begin{array}{l}\text { Finasteride, } \\
5 \mathrm{mg} \mathrm{OD}\end{array}$ & Placebo & $\mathrm{n} / \mathrm{a}$ & $\mathrm{n} / \mathrm{a}$ & 34 & 6 months & TPV, PSA & Secondary & High Risk \\
\hline $\begin{array}{l}\text { Marks 1997, } \\
{[43]}\end{array}$ & $\begin{array}{c}\text { Finasteride, } \\
5 \mathrm{mg} \mathrm{OD}\end{array}$ & Placebo & $\mathrm{n} / \mathrm{a}$ & $\mathrm{n} / \mathrm{a}$ & 41 & 6 months & TPV, PSA & Secondary & $\begin{array}{l}\text { Moderate } \\
\text { Risk }\end{array}$ \\
\hline $\begin{array}{l}\text { Gormley } \\
\text { 1992, [44] }\end{array}$ & $\begin{array}{c}\text { Finasteride, } \\
5 \mathrm{mg} \mathrm{OD}\end{array}$ & Placebo & $\mathrm{n} / \mathrm{a}$ & $\mathrm{n} / \mathrm{a}$ & 597 & 12 months & TPV, PSA & Secondary & $\begin{array}{l}\text { Moderate } \\
\text { Risk }\end{array}$ \\
\hline $\begin{array}{l}\text { Roehrborn } \\
\text { 2002, [45] }\end{array}$ & $\begin{array}{l}\text { Dutasteride, } \\
0.5 \mathrm{mg} \mathrm{OD}\end{array}$ & Placebo & $\mathrm{n} / \mathrm{a}$ & $\mathrm{n} / \mathrm{a}$ & 4325 & 24 months & $\begin{array}{l}\text { TPV, TZV, } \\
\text { PSA }\end{array}$ & Secondary & Low Risk \\
\hline $\begin{array}{l}\mathrm{Na} 2012, \\
{[46]}\end{array}$ & $\begin{array}{l}\text { Dutasteride, } \\
0.5 \mathrm{mg} \mathrm{OD}\end{array}$ & Placebo & $\mathrm{n} / \mathrm{a}$ & $\mathrm{n} / \mathrm{a}$ & 253 & 6 months & TPV, PSA & Primary & $\begin{array}{c}\text { Moderate } \\
\text { Risk }\end{array}$ \\
\hline $\begin{array}{l}\text { Tsukamoto } \\
\text { 2009, [47] }\end{array}$ & $\begin{array}{l}\text { Dutasteride, } \\
0.5 \mathrm{mg} \mathrm{OD}\end{array}$ & Placebo & $\mathrm{n} / \mathrm{a}$ & $\mathrm{n} / \mathrm{a}$ & 378 & 6 months & TPV, PSA & Secondary & $\begin{array}{c}\text { Moderate } \\
\text { Risk }\end{array}$ \\
\hline $\begin{array}{l}\text { Andriole } \\
2010,[48]\end{array}$ & $\begin{array}{l}\text { Dutasteride, } \\
0.5 \mathrm{mg} \mathrm{OD}\end{array}$ & Placebo & $\mathrm{n} / \mathrm{a}$ & $\mathrm{n} / \mathrm{a}$ & 8231 & 48 months & TPV & Secondary & $\begin{array}{c}\text { Moderate } \\
\text { Risk }\end{array}$ \\
\hline $\begin{array}{l}\text { Nickel 2011, } \\
\text { [49] }\end{array}$ & $\begin{array}{l}\text { Finasteride, } \\
5 \mathrm{mg} \mathrm{OD}\end{array}$ & $\begin{array}{l}\text { Dutasteride, } \\
0.5 \mathrm{mg} \mathrm{OD}\end{array}$ & $\mathrm{n} / \mathrm{a}$ & $\mathrm{n} / \mathrm{a}$ & 1630 & 12 months & TPV, PSA & Primary & $\begin{array}{l}\text { Moderate } \\
\text { Risk }\end{array}$ \\
\hline $\begin{array}{l}\text { Carraro } \\
1996,[50]\end{array}$ & $\begin{array}{c}\text { Finasteride, } \\
5 \mathrm{mg} \mathrm{OD}\end{array}$ & $\begin{array}{l}\text { Serenoa repens, } \\
320 \mathrm{mg} \text { OD }\end{array}$ & $\mathrm{n} / \mathrm{a}$ & $\mathrm{n} / \mathrm{a}$ & 1098 & 6 months & TPV, PSA & Secondary & Low Risk \\
\hline $\begin{array}{l}\text { Kuo 1998, } \\
{[51]}\end{array}$ & $\begin{array}{c}\text { Dibenyline, } \\
10 \text { mg BD }\end{array}$ & $\begin{array}{c}\text { Finasteride, } \\
5 \mathrm{mg} \mathrm{OD}\end{array}$ & $\mathrm{n} / \mathrm{a}$ & $\mathrm{n} / \mathrm{a}$ & 125 & 6 months & TPV & Secondary & High Risk \\
\hline $\begin{array}{l}\text { Jeong 2009, } \\
{[52]}\end{array}$ & $\begin{array}{l}\text { a blocker OD } \\
\text { plus } \\
\text { Finasteride, } \\
5 \text { mg OD }\end{array}$ & $\begin{array}{l}\text { a blocker OD } \\
\text { plus } \\
\text { Dutasteride, } \\
0.5 \text { mg OD }\end{array}$ & $\mathrm{n} / \mathrm{a}$ & $\mathrm{n} / \mathrm{a}$ & 120 & 24 months & TPV, PSA & Secondary & $\begin{array}{l}\text { Moderate } \\
\text { Risk }\end{array}$ \\
\hline $\begin{array}{l}\text { Pinggera } \\
2014,[53]\end{array}$ & $\begin{array}{l}\text { Tadalafil, } \\
5 \text { mg OD }\end{array}$ & Placebo & $\mathrm{n} / \mathrm{a}$ & $\mathrm{n} / \mathrm{a}$ & 97 & 8 weeks & $\begin{array}{c}\text { Perfusion } \\
\text { parameters }\end{array}$ & Primary & $\begin{array}{l}\text { Moderate } \\
\text { Risk }\end{array}$ \\
\hline $\begin{array}{l}\text { Morgia } \\
\text { 2018, [54] }\end{array}$ & $\begin{array}{c}\text { Serenoa } \\
\text { repens plus } \\
\text { Selenium, OD }\end{array}$ & $\begin{array}{l}\text { Tadalafil, } \\
5 \text { mg OD }\end{array}$ & $\mathrm{n} / \mathrm{a}$ & $\mathrm{n} / \mathrm{a}$ & 427 & 6 months & TPV, PSA & Secondary & $\begin{array}{l}\text { Moderate } \\
\text { Risk }\end{array}$ \\
\hline $\begin{array}{l}\text { Kosilov 2019, } \\
{[55]}\end{array}$ & $\begin{array}{l}\text { Tadalafil, } \\
5 \text { mg OD }\end{array}$ & $\begin{array}{l}\text { Tadalafil, } \\
5 \text { mg OD plus } \\
\text { Solifenacin, } \\
10 \text { mg OD }\end{array}$ & $\mathrm{n} / \mathrm{a}$ & $\mathrm{n} / \mathrm{a}$ & 214 & 12 months & TPV & Secondary & High Risk \\
\hline $\begin{array}{l}\text { Oztrurk } \\
\text { 2011, [56] }\end{array}$ & $\begin{array}{l}\text { Alfuzosin } \mathrm{XL} \\
\text { OD }\end{array}$ & $\begin{array}{l}\text { Alfuzosin XL OD } \\
\text { plus Sildenafil, } \\
50 \text { mg OD }\end{array}$ & $\mathrm{n} / \mathrm{a}$ & $\mathrm{n} / \mathrm{a}$ & 100 & 3 months & TPV, PSA & Secondary & High Risk \\
\hline $\begin{array}{l}\text { Joo 2012, } \\
{[57]}\end{array}$ & $\begin{array}{c}\text { Tamsulosin, } \\
0.2 \mathrm{mg} O D\end{array}$ & $\begin{array}{l}\text { Tamsulosin, } \\
0.2 \text { mg OD plus } \\
\text { Dutasteride, } \\
\text { 0.5 mg OD }\end{array}$ & $\mathrm{n} / \mathrm{a}$ & $\mathrm{n} / \mathrm{a}$ & 216 & 12 months & $\begin{array}{l}\text { TPV, TZV, } \\
\text { PSA }\end{array}$ & Secondary & High Risk \\
\hline $\begin{array}{l}\text { Choi 2016, } \\
{[58]}\end{array}$ & $\begin{array}{l}\text { Tamsulosin, } \\
0.2 \text { mg OD }\end{array}$ & $\begin{array}{l}\text { Tamsulosin, } \\
0.2 \text { mg OD plus } \\
\text { Dutasteride, } \\
0.5 \text { mg OD }\end{array}$ & $\mathrm{n} / \mathrm{a}$ & $\mathrm{n} / \mathrm{a}$ & 118 & 12 months & $\begin{array}{l}\text { TPV, TZV, } \\
\text { PSA }\end{array}$ & Secondary & Low Risk \\
\hline $\begin{array}{l}\text { Mohanty } \\
\text { 2006, [59] }\end{array}$ & $\begin{array}{l}\text { Tamsulosin, } \\
0.4 \text { mg OD plus } \\
\text { Finasteride, } \\
5 \text { mg OD }\end{array}$ & $\begin{array}{l}\text { Tamsulosin, } \\
0.4 \text { mg OD plus } \\
\text { Dutasteride, } \\
\text { 0.5 mg OD }\end{array}$ & $\mathrm{n} / \mathrm{a}$ & $\mathrm{n} / \mathrm{a}$ & 106 & 6 months & TPV, PSA & Secondary & High Risk \\
\hline
\end{tabular}




\begin{tabular}{|c|c|c|c|c|c|c|c|c|c|}
\hline $\begin{array}{c}\text { Study, } \\
\text { [reference] }\end{array}$ & $\begin{array}{l}\text { Comparator } 1, \\
\text { Daily dosage }\end{array}$ & $\begin{array}{l}\text { Comparator 2, } \\
\text { Daily dosage }\end{array}$ & $\begin{array}{l}\text { Comparator 3, } \\
\text { Daily dosage }\end{array}$ & $\begin{array}{l}\text { Comparator } 4 \text {, } \\
\text { Daily dosage }\end{array}$ & $\begin{array}{l}\text { No. subjects } \\
\text { randomized }\end{array}$ & $\begin{array}{l}\text { Duration of } \\
\text { Follow up }\end{array}$ & $\begin{array}{c}\text { Reported } \\
\text { parameters }\end{array}$ & $\begin{array}{l}\text { Primary } \\
\text { or Secondary } \\
\text { endpoints }\end{array}$ & $\begin{array}{c}\text { Study rating } \\
\text { based on } \\
\text { AHRQ criteria }\end{array}$ \\
\hline $\begin{array}{l}\text { Yamanishi } \\
\text { 2017, [60] }\end{array}$ & $\begin{array}{l}\text { Tamsulosin, } \\
0.2 \text { mg OD plus } \\
\text { Dutasteride, } \\
0.5 \text { mg OD }\end{array}$ & $\begin{array}{l}\text { Tamsulosin, } \\
0.2 \text { mg OD plus } \\
\text { Dutasteride, } \\
\text { 0.5 mg OD plus } \\
\text { imidafenacin, } \\
0.2 \mathrm{mg} \mathrm{OD}\end{array}$ & $\mathrm{n} / \mathrm{a}$ & $\mathrm{n} / \mathrm{a}$ & 163 & 24 weeks & TPV, PSA & Secondary & $\begin{array}{c}\text { Moderate } \\
\text { Risk }\end{array}$ \\
\hline $\begin{array}{l}\text { Ryu 2014, } \\
\text { [61] }\end{array}$ & $\begin{array}{l}\text { Tamsulosin, } \\
0.2 \text { mg OD }\end{array}$ & $\begin{array}{l}\text { Tamsulosin, } \\
0.2 \text { mg OD plus } \\
\text { Serenoa repens, } \\
320 \text { mg OD }\end{array}$ & $\mathrm{n} / \mathrm{a}$ & $\mathrm{n} / \mathrm{a}$ & 120 & 12 months & TPV, PSA & Secondary & $\begin{array}{l}\text { Moderate } \\
\text { Risk }\end{array}$ \\
\hline $\begin{array}{l}\text { Argirovic } \\
2013,[62]\end{array}$ & $\begin{array}{l}\text { Tamsulosin, } \\
0.4 \text { mg OD }\end{array}$ & $\begin{array}{l}\text { Serenoa repens, } \\
320 \text { mg OD }\end{array}$ & $\begin{array}{l}\text { Tamsulosin, } \\
0.4 \text { mg OD plus } \\
\text { Serenoa repens, } \\
320 \text { mg OD }\end{array}$ & $\mathrm{n} / \mathrm{a}$ & 184 & 6 months & TPV, PSA & Secondary & High Risk \\
\hline $\begin{array}{l}\text { Beiraghdar } \\
\text { 2017, [63] }\end{array}$ & $\begin{array}{l}\text { Phytotherapy } \\
\text { (Non-Sr) }\end{array}$ & Placebo & $\mathrm{n} / \mathrm{a}$ & $\mathrm{n} / \mathrm{a}$ & 86 & 2 weeks & TPV & Secondary & $\begin{array}{c}\text { Moderate } \\
\text { Risk }\end{array}$ \\
\hline $\begin{array}{l}\text { Berges 1995, } \\
{[64]}\end{array}$ & $\begin{array}{l}\text { Phytotherapy } \\
\text { (Non-Sr) }\end{array}$ & Placebo & $\mathrm{n} / \mathrm{a}$ & $\mathrm{n} / \mathrm{a}$ & 163 & 6 months & TPV & Secondary & $\begin{array}{c}\text { Moderate } \\
\text { Risk }\end{array}$ \\
\hline $\begin{array}{l}\text { Safarinejad } \\
2005,[65]\end{array}$ & $\begin{array}{l}\text { Phytotherapy } \\
\text { (Non-Sr) }\end{array}$ & Placebo & $\mathrm{n} / \mathrm{a}$ & $\mathrm{n} / \mathrm{a}$ & 620 & 6 months & TPV, PSA & Secondary & High Risk \\
\hline $\begin{array}{l}\text { Bent 2006, } \\
{[66]}\end{array}$ & $\begin{array}{c}\text { Serenoa } \\
\text { repens, } \\
160 \text { mg BD }\end{array}$ & Placebo & $\mathrm{n} / \mathrm{a}$ & $\mathrm{n} / \mathrm{a}$ & 225 & 12 months & $\begin{array}{l}\text { TPV, TZV, } \\
\text { PSA }\end{array}$ & Secondary & Low Risk \\
\hline $\begin{array}{l}\text { Marks 2000, } \\
{[67]}\end{array}$ & $\begin{array}{l}\text { Serenoa } \\
\text { repens }\end{array}$ & Placebo & $\mathrm{n} / \mathrm{a}$ & $\mathrm{n} / \mathrm{a}$ & 44 & 24 weeks & $\begin{array}{l}\text { TPV, TZV, } \\
\text { PSA }\end{array}$ & Secondary & $\begin{array}{c}\text { Moderate } \\
\text { Risk }\end{array}$ \\
\hline Ye 2019, [68] & $\begin{array}{c}\text { Serenoa } \\
\text { repens, } \\
320 \text { mg OD }\end{array}$ & Placebo & $\mathrm{n} / \mathrm{a}$ & $\mathrm{n} / \mathrm{a}$ & 325 & 24 weeks & TPV, PSA & Secondary & Low Risk \\
\hline $\begin{array}{l}\text { Zhang 2008, } \\
\text { [69] }\end{array}$ & $\begin{array}{l}\text { Phytotherapy } \\
\text { (Non-Sr) }\end{array}$ & Placebo & $\mathrm{n} / \mathrm{a}$ & $\mathrm{n} / \mathrm{a}$ & 49 & 4 months & TPV & Secondary & High Risk \\
\hline $\begin{array}{l}\text { Shi 2008, } \\
{[70]}\end{array}$ & $\begin{array}{l}\text { Serenoa } \\
\text { repens }\end{array}$ & Placebo & $\mathrm{n} / \mathrm{a}$ & $\mathrm{n} / \mathrm{a}$ & 94 & 12 weeks & TPV, PSA & Secondary & $\begin{array}{c}\text { Moderate } \\
\text { Risk }\end{array}$ \\
\hline $\begin{array}{l}\text { Guzman } \\
\text { 2019, [71] }\end{array}$ & $\begin{array}{l}\text { Phytotherapy } \\
\text { (Non-Sr), OD }\end{array}$ & $\begin{array}{l}\text { Terazosin, } \\
5 \mathrm{mg} \mathrm{OD}\end{array}$ & $\mathrm{n} / \mathrm{a}$ & $\mathrm{n} / \mathrm{a}$ & 100 & 6 months & TPV & Secondary & $\begin{array}{c}\text { Moderate } \\
\text { Risk }\end{array}$ \\
\hline $\begin{array}{l}\text { Braeckman } \\
\text { 1997, [72] }\end{array}$ & $\begin{array}{c}\text { Serenoa } \\
\text { repens, } \\
320 \text { mg OD }\end{array}$ & $\begin{array}{l}\text { Serenoa repens, } \\
160 \text { mg OD }\end{array}$ & $\mathrm{n} / \mathrm{a}$ & $\mathrm{n} / \mathrm{a}$ & 84 & 12 months & TPV & Secondary & High Risk \\
\hline $\begin{array}{l}\text { Allott 2019, } \\
\text { [73] }\end{array}$ & Statin users & $\begin{array}{c}\text { Non- Statin } \\
\text { users }\end{array}$ & $\mathrm{n} / \mathrm{a}$ & $\mathrm{n} / \mathrm{a}$ & 4106 & 48 months & TPV & Primary & $\begin{array}{l}\text { Moderate } \\
\text { Risk }\end{array}$ \\
\hline $\begin{array}{l}\text { Mills 2007, } \\
{[74]}\end{array}$ & $\begin{array}{l}\text { Atorvastatin, } \\
80 \text { mg OD }\end{array}$ & Placebo & $\mathrm{n} / \mathrm{a}$ & $\mathrm{n} / \mathrm{a}$ & 350 & 26 weeks & $\begin{array}{l}\text { TPV, TZV, } \\
\text { PSA }\end{array}$ & Secondary & Low Risk \\
\hline $\begin{array}{l}\text { Zhang 2015, } \\
\text { [75] }\end{array}$ & $\begin{array}{l}\text { Atorvastatin, } \\
20 \text { mg OD }\end{array}$ & Placebo & $\mathrm{n} / \mathrm{a}$ & $\mathrm{n} / \mathrm{a}$ & 81 & 12 months & TPV, PSA & Secondary & $\begin{array}{c}\text { Moderate } \\
\text { Risk }\end{array}$ \\
\hline $\begin{array}{l}\text { Safwat 2018, } \\
{[76]}\end{array}$ & $\begin{array}{l}\text { Tamsulosin, } \\
0.4 \text { mg OD }\end{array}$ & $\begin{array}{l}\text { Tamsulosin, } \\
0.4 \text { mg OD plus } \\
\text { Cholecalciferol } \\
\text { 600IU OD }\end{array}$ & $\mathrm{n} / \mathrm{a}$ & $\mathrm{n} / \mathrm{a}$ & 389 & 24 months & TPV, PSA & Secondary & $\begin{array}{c}\text { Moderate } \\
\text { Risk }\end{array}$ \\
\hline $\begin{array}{l}\text { Ghadian } \\
\text { 2017, [77] }\end{array}$ & $\begin{array}{c}\Omega 3300 \mathrm{mg} \\
\text { plus } \\
\text { Tamsulosin } \\
0.4 \mathrm{mg} \text { plus } \\
\text { Finasteride } \\
5 \mathrm{mg}\end{array}$ & $\begin{array}{l}\text { Tamsulosin } \\
0.4 \text { mg plus } \\
\text { Finasteride } \\
5 \mathrm{mg}\end{array}$ & $\mathrm{n} / \mathrm{a}$ & $\mathrm{n} / \mathrm{a}$ & 100 & 6 months & TPV & Secondary & High Risk \\
\hline $\begin{array}{l}\text { Di Silverio } \\
2005,[78]\end{array}$ & $\begin{array}{c}\text { Finasteride, } \\
5 \mathrm{mg} \text { OD }\end{array}$ & $\begin{array}{l}\text { Finasteride, } \\
5 \text { mg OD plus } \\
\text { Rofecoxib, } \\
25 \text { mg OD }\end{array}$ & $\mathrm{n} / \mathrm{a}$ & $\mathrm{n} / \mathrm{a}$ & 46 & 6 months & TPV, PSA & Secondary & $\begin{array}{l}\text { Moderate } \\
\text { Risk }\end{array}$ \\
\hline
\end{tabular}


Table 1. Continued

\begin{tabular}{|c|c|c|c|c|c|c|c|c|c|}
\hline $\begin{array}{c}\text { Study, } \\
\text { [reference] }\end{array}$ & $\begin{array}{c}\text { Comparator } 1 \\
\text { Daily dosage }\end{array}$ & $\begin{array}{l}\text { Comparator 2, } \\
\text { Daily dosage }\end{array}$ & $\begin{array}{l}\text { Comparator } 3 \\
\text { Daily dosage }\end{array}$ & $\begin{array}{l}\text { Comparator } 4, \\
\text { Daily dosage }\end{array}$ & $\begin{array}{l}\text { No. subjects } \\
\text { randomized }\end{array}$ & $\begin{array}{l}\text { Duration of } \\
\text { Follow up }\end{array}$ & $\begin{array}{l}\text { Reported } \\
\text { parameters }\end{array}$ & $\begin{array}{l}\text { Primary } \\
\text { or Secondary } \\
\text { endpoints }\end{array}$ & $\begin{array}{l}\text { Study rating } \\
\text { based on } \\
\text { AHRQ criteria }\end{array}$ \\
\hline $\begin{array}{l}\text { Goodarzt } \\
2011,[79]\end{array}$ & $\begin{array}{l}\text { Terazosin, } \\
2 \text { mg OD }\end{array}$ & $\begin{array}{c}\text { Terazosin, } \\
2 \text { mg OD plus } \\
\text { Celecoxib, } \\
200 \text { mg OD }\end{array}$ & $\mathrm{n} / \mathrm{a}$ & $\mathrm{n} / \mathrm{a}$ & 160 & 12 weeks & TPV, PSA & Secondary & High Risk \\
\hline $\begin{array}{l}\text { Jhang 2013, } \\
\text { [80] }\end{array}$ & $\begin{array}{l}\text { Doxazosin, } \\
4 \text { mg OD }\end{array}$ & $\begin{array}{c}\text { Doxazosin, } \\
4 \text { mg OD plus } \\
\text { Celecoxib, } \\
200 \text { mg OD }\end{array}$ & $\mathrm{n} / \mathrm{a}$ & $\mathrm{n} / \mathrm{a}$ & 122 & 3 months & TPV, PSA & Secondary & High Risk \\
\hline $\begin{array}{l}\text { Page 2011, } \\
\text { [81] }\end{array}$ & $\begin{array}{l}\text { Testosterone } \\
1 \% 7.5 \mathrm{mg} \text { OD } \\
\text { plus placebo }\end{array}$ & $\begin{array}{c}\text { Testosterone } 1 \% \\
7.5 \mathrm{mg} \text { OD plus } \\
\text { Dutasteride, } \\
0.5 \mathrm{mg} \mathrm{OD}\end{array}$ & $n / a$ & $\mathrm{n} / \mathrm{a}$ & 53 & 6 months & TPV, PSA & Secondary & $\begin{array}{l}\text { Moderate } \\
\text { Risk }\end{array}$ \\
\hline $\begin{array}{l}\text { Kacker 2014, } \\
{[82]}\end{array}$ & $\begin{array}{l}\text { Testosterone } \\
\text { plus placebo }\end{array}$ & $\begin{array}{l}\text { Testosterone } \\
\text { plus } \\
\text { Dutasteride, } \\
0.5 \mathrm{mg} \mathrm{OD}\end{array}$ & $\mathrm{n} / \mathrm{a}$ & $\mathrm{n} / \mathrm{a}$ & 23 & 12 months & TPV, PSA & Primary & $\begin{array}{l}\text { Moderate } \\
\text { Risk }\end{array}$ \\
\hline $\begin{array}{l}\text { Chung 2011, } \\
\text { [83] }\end{array}$ & $\begin{array}{l}\text { a blocker OD } \\
\text { plus 5ARI }\end{array}$ & $\begin{array}{c}\text { a blocker OD } \\
\text { plus } \\
\text { 5ARI plus } \\
\text { Tolterodine }\end{array}$ & $n / a$ & $\mathrm{n} / \mathrm{a}$ & 137 & 12 months & $\begin{array}{l}\text { TPV, TZI, } \\
\text { PSA }\end{array}$ & Secondary & $\begin{array}{l}\text { Moderate } \\
\text { Risk }\end{array}$ \\
\hline
\end{tabular}

AHRQ - Agency for Healthcare Research and Quality; BD - Twice Daily; $\mathrm{n} / \mathrm{a}$ - not applicable; Non-Sr - other than Serenoa repens; OD - once daily; PSA - prostate-specific antigen; $\mathrm{Sr}$ - Serenoa repens; TPV - total prostate volume; TZI - transitional zone index; TZV - transitional zone volume

in 10 comparisons, while 9 comparisons assessed the effect of non-urological medications. Among them, only 10 trials were powered to assess changes in morphometric parameters, while 57 reported a morphometric parameter change as secondary outcome. The characteristics of included RCTs are presented in Table 1.

\section{Assessment of study quality}

The summary of RoB assessment is presented in Figure 2 and Figure 3. Based on AHRQ criteria, 16 RCTs were graded as low-risk, 31 as moderaterisk and 20 as high-risk (Table 2).

\section{Data Synthesis}

\section{a1-blockers}

Six trials randomized men $(\mathrm{n}=4525)$ to $\alpha$-blocker versus placebo (Table 1) [18-23]. The MTOPS randomized men to receive doxazosin, finasteride, combination or placebo and reported $+24 \%(+10.1 \mathrm{ml})$ change in TPV of patients receiving doxazosin at 4 years, similar to placebo $(+24 \%$ or $+8.8 \mathrm{ml})$ [19]. The Veteran Affairs Cooperative Study (VACOOP Study) reported similar changes in terazosin and placebo arms at 12 months $(+2.0 \%$ or $+0.5 \mathrm{ml}$ vs $+2.3 \%$ or $+0.5 \mathrm{ml}$ ) [18]. The ALFUS trial reported non-significant changes from baseline at 3 months in men who received alfuzosin or placebo in both TPV (-2\% or $0.25 \mathrm{ml} \mathrm{vs}+3 \%$ or $+0.46 \mathrm{ml})$ and TZV ( $-2 \%$ vs $-5 \%$ or $-0.8 \mathrm{ml}$ vs $-0.39 \mathrm{ml}$ ) [22]. Five RCTs reported on post treatment PSA changes, which were similar to placebo [18-21, 23]. There was no information on prostate perfusion parameters.

Ten RCTs randomized men $(\mathrm{n}=5479)$ to an $\alpha$-blocker versus an active comparator with a follow-up to 24 weeks [24-33]. All studies reported non-significant TPV changes from baseline $(-3.4 \%$ to $+9.5 \%$ or $-1.4 \mathrm{ml}$ to $+6.32 \mathrm{ml}$ ). CombAT randomized men to receive tamsulosin, dutasteride or combination and followed them up for 4.5 years [32]. Men in the tamsulosin arm increased TPV by $+4.6 \%(+2.57 \mathrm{ml})$ and TZV by $+18.2 \%$ ( $+5.5 \mathrm{ml})$. A single trial compared tamsulosin to silodosin and reported a reduction of TPV after 6 months, which was greater in the silodosin arm (-2.8\% vs $-8.6 \%$ or $-1.0 \mathrm{ml}$ vs $-3.6 \mathrm{ml}, \mathrm{p}=0.594)$ [27]. TPV changes after 3-months of Naftopidil treatment were negligible and comparable to tamsulosin [99]. A trial with high $\mathrm{RoB}$ reported $+9.5 \%(+6.32 \mathrm{ml})$ increase in TPV after 6 months tamsulosin monotherapy, which was neither significantly different from baseline $(p=0.17)$ nor from the comparator [30]. The Alfin study reported no significant change in TPV $(-1 \%$ or $-0.2 \mathrm{ml})$ or PSA value $(+3.3 \%$ or $+0.1 \mathrm{ng} / \mathrm{dl})$ after 6 months of alfuzosin treatment [33]. PSA was reported unchanged in four tamsulosin studies (-5.0\% to $+7.4 \%$ ) $[24,28,29,31]$. Tamsulosin monotherapy enhanced prostate perfusion $(+146 \%)$ as opposed to 
Central European JOURnAL of UROLOGY

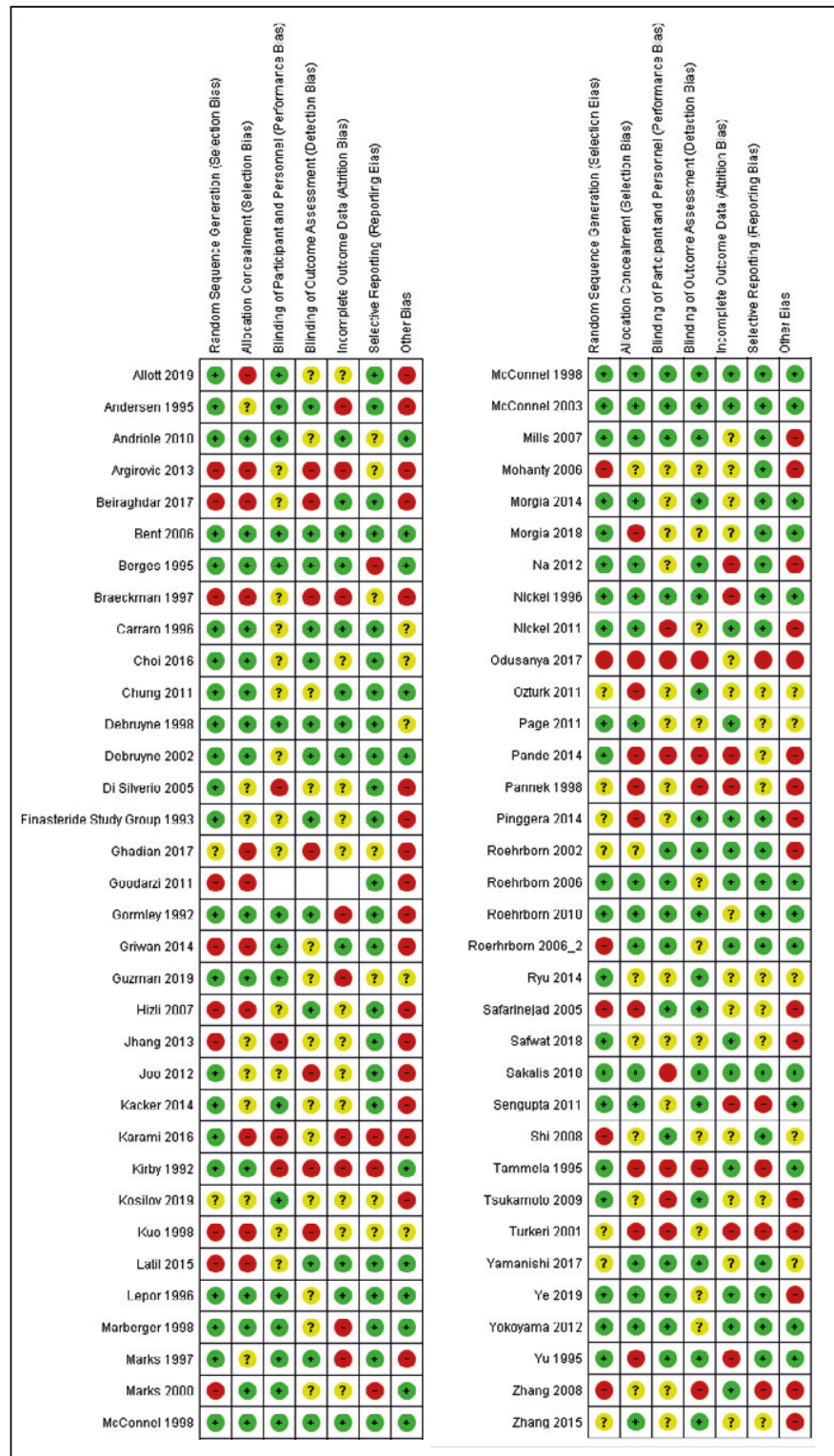

Figure 2. The risk of bias summary.

tamsulosin and solifenacin combination treatment $(-41 \%)$ in a male overactive bladder $(\mathrm{OAB})$ cohort [34].

\section{5-ARIs}

Sixteen trials randomized men $(\mathrm{n}=21109)$ to 5 -ARI versus placebo (Table 1) [18, 19, 35-48]. Twelve finasteride trials reported significant changes in TPV as compared to baseline and to placebo [18, 19, 35-44]. The quantitative synthesis revealed a large effect size in favor of finasteride [SMD]: -1.15 (95\%CI: -1.26 to $-1.04, \mathrm{p}<0.001$ ) (Figure 2). The effect on TPV varies between studies with different follow-ups. Trials with 3-6 months' follow-up report changes between $-4.8 \%$ and $-26.1 \%$, while trials with follow-up

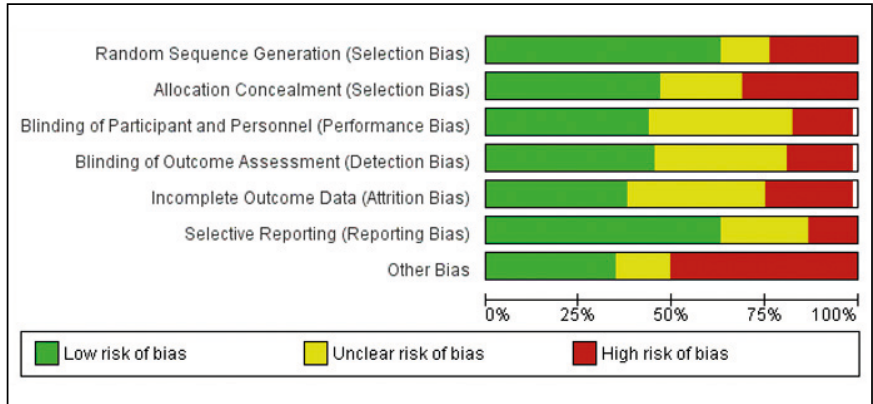

Figure 3. The risk of bias graph.

of 12 months or longer report higher TPV changes $(-15.3 \%$ to $-22.4 \%$ or $-8.1 \mathrm{ml}$ to $-10.53 \mathrm{ml})$. The finasteride study group randomized men to finasteride $1 \mathrm{mg}$ versus finasteride $5 \mathrm{mg}$ versus placebo, and reported similar TPV changes at 12 months (-23.6\% vs $-22.4 \%$ vs $-5 \%$ ), but the later was superior in improvement of clinical parameters such as maximum flow rate and relevant questionnaires scores [40].

Four dutasteride trials reported significant changes in TPV both from baseline or as compared to placebo [45-48]. The quantitative analysis revealed a large size effect [SMD]: -0.66 (95\%CI: -0.83 to -0.49 , $\mathrm{p}<0.001$ ) (Figure 4). The effect on TPV appears homogenous among studies with different followup and ranges between $-17.5 \%$ and $-27.0 \%(-7.2 \mathrm{ml}$ to $-13.6 \mathrm{ml})$. Dutasteride also significantly reduces TZV (-20.1\% or $-7.1 \mathrm{ml}, \mathrm{p}<0.001)$, an effect which is evident from the first month of treatment.

Nine finasteride RCTs report significant changes in PSA as compared to baseline or to placebo [18, $19,35,36,39,40,42,43,44]$. The quantitative analysis revealed a moderate size effect in favor of finasteride ([SMD]:-0.63, 95\%CI:-0.76 to 0.51), $\mathrm{p}<0.001$ ) (Figure 4). Trials with 12 months followup or more report a PSA change of $-46.0 \%$ to $-52 \%$. Three dutasteride RCTs, report significant reduction in PSA (-42.2\% to $-52.4 \%)$, compared to both baseline $(\mathrm{p}<0.05)$ and placebo $(\mathrm{p}<0.05)[45,46,47]$.

Five RCTs randomized men $(\mathrm{n}=3615)$ to finasteride versus an active comparator. All studies report significant TPV changes from baseline $(-10.5 \%$ to $-24.3 \%$ or $-4.3 \mathrm{ml}$ to $-7.5 \mathrm{ml}$ ) and significant difference from the active comparator [30, 33, 49, 50, 51]. The dutasteride arm of CombAT reported $-28.0 \%$ $(-15.3 \mathrm{ml})$ and $-26.5 \%(-8.03 \mathrm{ml})$ reduction of TPV and TZV, respectively [32]. The EPICS study randomized men to finasteride or dutasteride for 12 months and found significant change from baseline in both arms $(-26.7 \%$ vs $-26.3 \%$ or $-13.99 \mathrm{ml}$ vs $-14.2 \mathrm{ml})$ without intergroup difference $(\mathrm{p}=0.65)$ [49]. Another trial reported similar changes after 12 months' treatment with finasteride or dutas- 
Table 2. Detailed rating for included trials based on criteria developed by the Agency for Healthcare Research and Quality (AHRQ). The ratings were 'Low-risk', 'Moderate-risk' or 'High-risk'

\begin{tabular}{|c|c|c|c|c|c|c|c|c|c|c|c|c|c|c|}
\hline \multirow{2}{*}{ Study } & \multicolumn{12}{|c|}{ Individual Quality Assessment Criteria Ratings } & \multirow{2}{*}{$\begin{array}{l}\text { Overall } \\
\text { Rating }\end{array}$} & \multirow{2}{*}{$\begin{array}{c}\text { COI } \\
\text { Absent? }\end{array}$} \\
\hline & $1 a$ & $1 \mathrm{~b}$ & $1 \mathrm{c}$ & $2 a$ & $2 b$ & $3 a$ & $3 b$ & $3 c$ & $3 d$ & $3 e$ & 4 & 5 & & \\
\hline Lepor 1996, [18] & $L R$ & $L R$ & $L R$ & UR & $\mathrm{LR}$ & $\mathrm{LR}$ & UR & $L R$ & $L R$ & $L R$ & LR & $L R$ & Low Risk & No \\
\hline McConnell 2003, [19] & $L R$ & $L R$ & $L R$ & UR & $L R$ & LR & UR & $L R$ & $L R$ & $L R$ & $L R$ & $L R$ & Low Risk & No \\
\hline Yokoyama 2012, [20] & $L R$ & UR & LR & UR & LR & $\mathrm{HR}$ & UR & $L R$ & $L R$ & $L R$ & $L R$ & $L R$ & Low Risk & No \\
\hline Roerhborn 2006, [21] & $L R$ & UR & $L R$ & $L R$ & $L R$ & UR & UR & $L R$ & $L R$ & $L R$ & $L R$ & $L R$ & Low Risk & No \\
\hline Roerhborn 2006, [22] & $\mathrm{HR}$ & $\mathrm{HR}$ & $L R$ & $L R$ & $L R$ & UR & $L R$ & $L R$ & $L R$ & $\mathrm{LR}$ & $L R$ & $L R$ & $\begin{array}{l}\text { Moderate } \\
\text { Risk }\end{array}$ & No \\
\hline Turkeri 2001, [23] & UR & UR & $H R$ & $H R$ & $L R$ & UR & UR & UR & $L R$ & $L R$ & $H R$ & $H R$ & High Risk & Unclear \\
\hline Debruyne 2002, [24] & LR & LR & LR & UR & LR & LR & LR & UR & $L R$ & $L R$ & $L R$ & $L R$ & Low Risk & No \\
\hline Sengupta 2011, [25] & $L R$ & $L R$ & $L R$ & UR & UR & LR & UR & UR & $L R$ & $L R$ & $H R$ & $H R$ & $\begin{array}{l}\text { Moderate } \\
\text { Risk }\end{array}$ & No \\
\hline Latil 2015, [26] & $\mathrm{HR}$ & $\mathrm{HR}$ & $L R$ & UR & $L R$ & UR & UR & UR & $L R$ & $L R$ & $L R$ & $L R$ & High Risk & Unclear \\
\hline Pande 2014, [27] & $L R$ & $\mathrm{HR}$ & $L R$ & $L R$ & $\mathrm{HR}$ & $H R$ & UR & $L R$ & $L R$ & $L R$ & $\mathrm{HR}$ & UR & $\begin{array}{l}\text { Moderate } \\
\text { Risk }\end{array}$ & Unclear \\
\hline Karami 2016, [28] & LR & $\mathrm{HR}$ & $H R$ & UR & $H R$ & UR & $H R$ & $H R$ & $L R$ & $L R$ & $H R$ & $H R$ & High Risk & Unclear \\
\hline Hizli 2007, [29] & $\mathrm{HR}$ & $\mathrm{HR}$ & $\mathrm{HR}$ & UR & $L R$ & $H R$ & $H R$ & UR & $L R$ & $L R$ & UR & $L R$ & High Risk & Unclear \\
\hline Odusanya 2017, [30] & $H R$ & $\mathrm{HR}$ & LR & $\mathrm{HR}$ & UR & $\mathrm{HR}$ & $\mathrm{HR}$ & $\mathrm{HR}$ & $L R$ & $L R$ & UR & $H R$ & High Risk & Unclear \\
\hline Morgia 2014, [31] & LR & LR & LR & UR & LR & LR & UR & LR & $L R$ & $L R$ & UR & $L R$ & Low Risk & Unclear \\
\hline Roehrborn 2010, [32] & $L R$ & $L R$ & $L R$ & $L R$ & UR & UR & UR & $L R$ & $L R$ & $L R$ & $L R$ & $L R$ & Low Risk & Unclear \\
\hline Debruyne 1998, [33] & $L R$ & $L R$ & $L R$ & UR & $L R$ & $L R$ & UR & $L R$ & $L R$ & $L R$ & $L R$ & $L R$ & Low Risk & Unclear \\
\hline Sakalis 2018, [34] & $L R$ & LR & $L R$ & $\mathrm{HR}$ & $\mathrm{HR}$ & $\mathrm{LR}$ & $\mathrm{LR}$ & $\mathrm{HR}$ & $L R$ & $L R$ & LR & $L R$ & $\begin{array}{l}\text { Moderate } \\
\text { Risk }\end{array}$ & Yes \\
\hline Andersen 1995, [35] & $L R$ & UR & $L R$ & $L R$ & $L R$ & UR & UR & $L R$ & $L R$ & $L R$ & $\mathrm{HR}$ & $\mathrm{LR}$ & $\begin{array}{l}\text { Moderate } \\
\text { Risk }\end{array}$ & No \\
\hline Nickel 1996, [36] & $L R$ & $L R$ & UR & $L R$ & $L R$ & $L R$ & $L R$ & $L R$ & $L R$ & $\mathrm{LR}$ & $\mathrm{HR}$ & $L R$ & Low Risk & No \\
\hline McConnel 1998, [37] & $L R$ & $L R$ & $L R$ & $L R$ & $L R$ & $L R$ & $L R$ & $L R$ & $L R$ & $L R$ & $L R$ & $L R$ & Low Risk & No \\
\hline Marberger 1998, [38] & $\mathrm{LR}$ & UR & $\mathrm{LR}$ & UR & $L R$ & $L R$ & $L R$ & $L R$ & $L R$ & LR & $\mathrm{HR}$ & LR & $\begin{array}{l}\text { Moderate } \\
\text { Risk }\end{array}$ & No \\
\hline Kirby 1992, [39] & $L R$ & UR & UR & $\mathrm{HR}$ & $\mathrm{HR}$ & $H R$ & $H R$ & UR & $L R$ & $L R$ & $L R$ & $\mathrm{HR}$ & High Risk & Unclear \\
\hline Finasteride group 1993, [40] & $L R$ & UR & UR & UR & UR & UR & $H R$ & $H R$ & $L R$ & LR & UR & $L R$ & $\begin{array}{l}\text { Moderate } \\
\text { Risk }\end{array}$ & No \\
\hline Tammela 1995, [41] & $L R$ & $H R$ & UR & $H R$ & $H R$ & UR & $H R$ & $H R$ & $L R$ & $L R$ & $L R$ & $H R$ & High Risk & Unclear \\
\hline Pannek 1998, [42] & UR & $H R$ & $H R$ & UR & UR & UR & UR & UR & $L R$ & LR & HR & UR & High Risk & Unclear \\
\hline Marks 1997, [43] & $\mathrm{LR}$ & UR & UR & $\mathrm{LR}$ & UR & UR & UR & UR & $L R$ & $\mathrm{LR}$ & $H R$ & $L R$ & $\begin{array}{c}\text { Moderate } \\
\text { Risk }\end{array}$ & Unclear \\
\hline Gormley 1992, [44] & $L R$ & LR & UR & UR & UR & $L R$ & $L R$ & $L R$ & $L R$ & $\mathrm{LR}$ & $H R$ & $L R$ & $\begin{array}{l}\text { Moderate } \\
\text { Risk }\end{array}$ & Unclear \\
\hline Roehrborn 2002, [45] & UR & UR & UR & $L R$ & $L R$ & $L R$ & $L R$ & $L R$ & $L R$ & $L R$ & $L R$ & $L R$ & Low Risk & Unclear \\
\hline Na 2012, [46] & $L R$ & $L R$ & UR & UR & UR & $L R$ & $L R$ & $L R$ & $L R$ & $L R$ & $L R$ & LR & $\begin{array}{c}\text { Moderate } \\
\text { Risk }\end{array}$ & Unclear \\
\hline Tsukamoto 2009, [47] & $L R$ & UR & UR & $\mathrm{HR}$ & $\mathrm{HR}$ & UR & UR & UR & $L R$ & $L R$ & UR & UR & $\begin{array}{l}\text { Moderate } \\
\text { Risk }\end{array}$ & No \\
\hline Andriole 2010, [48] & $L R$ & LR & UR & UR & UR & UR & $\mathrm{LR}$ & UR & $L R$ & $L R$ & $\mathrm{LR}$ & UR & $\begin{array}{l}\text { Moderate } \\
\text { Risk }\end{array}$ & No \\
\hline Nickel 2011, [49] & $L R$ & $L R$ & UR & $H R$ & UR & UR & $L R$ & UR & $L R$ & $L R$ & $L R$ & LR & $\begin{array}{c}\text { Moderate } \\
\text { Risk }\end{array}$ & No \\
\hline Carraro 1996, [50] & $L R$ & $L R$ & $L R$ & UR & $L R$ & $L R$ & UR & UR & $L R$ & $L R$ & $L R$ & $L R$ & Low Risk & Unclear \\
\hline Kuo $1998,[51]$ & $\mathrm{HR}$ & UR & UR & $H R$ & UR & UR & $L R$ & LR & $L R$ & $L R$ & UR & $H R$ & High Risk & Unclear \\
\hline Jeong 2009, [52] & UR & UR & $L R$ & $L R$ & UR & UR & UR & UR & $L R$ & $L R$ & $L R$ & UR & $\begin{array}{l}\text { Moderate } \\
\text { risk }\end{array}$ & Unclear \\
\hline
\end{tabular}


Table 2. Continued

\begin{tabular}{|c|c|c|c|c|c|c|c|c|c|c|c|c|c|c|}
\hline \multirow{2}{*}{ Study } & \multicolumn{12}{|c|}{ Individual Quality Assessment Criteria Ratings } & \multirow{2}{*}{$\begin{array}{l}\text { Overall } \\
\text { Rating }\end{array}$} & \multirow{2}{*}{$\begin{array}{c}\text { COI } \\
\text { Absent? }\end{array}$} \\
\hline & $1 a$ & $1 b$ & 1c & $2 a$ & $2 b$ & $3 a$ & $3 b$ & $3 c$ & $3 d$ & $3 e$ & 4 & 5 & & \\
\hline Jeong 2009, [52] & UR & UR & $L R$ & LR & UR & UR & UR & UR & $\mathrm{LR}$ & LR & LR & UR & $\begin{array}{l}\text { Moderate } \\
\text { risk }\end{array}$ & Unclear \\
\hline Pinggera 2014, [53] & UR & UR & LR & $L R$ & LR & UR & UR & UR & $\mathrm{LR}$ & LR & $L R$ & LR & $\begin{array}{c}\text { Moderate } \\
\text { Risk }\end{array}$ & No \\
\hline Morgia 2018, [54] & LR & $\mathrm{HR}$ & $\mathrm{LR}$ & UR & LR & UR & UR & UR & LR & LR & UR & $L R$ & $\begin{array}{c}\text { Moderate } \\
\text { Risk }\end{array}$ & No \\
\hline Kosilov 2019, [55] & UR & UR & $H R$ & LR & UR & UR & UR & UR & LR & LR & UR & UR & High Risk & Unclear \\
\hline Ozturk 2011, [56] & UR & $H R$ & $L R$ & UR & UR & LR & $H R$ & UR & $L R$ & $L R$ & UR & UR & High Risk & Unclear \\
\hline Joo 2012, [57] & $L R$ & UR & UR & UR & $\mathrm{HR}$ & UR & UR & $L R$ & $L R$ & $L R$ & UR & UR & High risk & Unclear \\
\hline Choi 2016, [58] & $L R$ & $L R$ & UR & LR & UR & UR & UR & $L R$ & LR & LR & LR & UR & Low Risk & Yes \\
\hline Mohanty 2006, [59] & $\mathrm{HR}$ & UR & UR & UR & UR & UR & UR & UR & $L R$ & $L R$ & UR & UR & High Risk & Unclear \\
\hline Yamanishi 2017, [60] & UR & LR & $\mathrm{LR}$ & LR & LR & LR & $L R$ & UR & LR & LR & UR & LR & $\begin{array}{c}\text { Moderate } \\
\text { Risk }\end{array}$ & No \\
\hline Ryu 2014, [61] & LR & UR & UR & UR & UR & UR & UR & UR & $\mathrm{LR}$ & $L R$ & UR & UR & $\begin{array}{l}\text { Moderate } \\
\text { Risk }\end{array}$ & Unclear \\
\hline Argirovic 2013, [62] & $H G$ & $\mathrm{HG}$ & $H R$ & UR & UR & $\mathrm{HR}$ & UR & $\mathrm{HR}$ & $L R$ & $L R$ & $\mathrm{HR}$ & UR & High Risk & Unclear \\
\hline Beiraghdar, 2017 [63] & $\mathrm{HR}$ & $\mathrm{HR}$ & LR & LR & UR & $H R$ & UR & LR & LR & LR & $L R$ & LR & $\begin{array}{l}\text { Moderate } \\
\text { risk }\end{array}$ & Yes \\
\hline Berges, 1995 [64] & $L R$ & $L R$ & LR & $L R$ & $L R$ & UR & UR & LR & $L R$ & LR & UR & $L R$ & $\begin{array}{c}\text { Moderate } \\
\text { risk }\end{array}$ & No \\
\hline Safarinejad, 2005 [65] & $\mathrm{HR}$ & $\mathrm{HR}$ & LR & UR & $L R$ & $L R$ & $L R$ & UR & LR & LR & UR & UR & High Risk & Yes \\
\hline Bent, 1995 [66] & $L R$ & $L R$ & $L R$ & $L R$ & UR & $L R$ & UR & $L R$ & $L R$ & $L R$ & $L R$ & UR & Low Risk & Unclear \\
\hline Marks, 2000 [67] & $L R$ & LR & $H R$ & $L R$ & LR & LR & UR & $\mathrm{HR}$ & LR & LR & UR & $\mathrm{HR}$ & $\begin{array}{l}\text { Moderate } \\
\text { risk }\end{array}$ & Unclear \\
\hline Ye, 2019 [68] & $L R$ & $L R$ & $L R$ & UR & $L R$ & $L R$ & UR & $L R$ & $L R$ & $L R$ & $L R$ & $L R$ & Low Risk & No \\
\hline Zhang 2008, [69] & $H R$ & UR & $L R$ & UR & UR & $L R$ & $H R$ & $H R$ & $L R$ & $L R$ & $L R$ & $H R$ & High Risk & Unclear \\
\hline Shi, 2008, [70] & $L R$ & $L R$ & $H R$ & $L R$ & LR & $L R$ & UR & UR & $L R$ & LR & UR & LR & $\begin{array}{c}\text { Moderate } \\
\text { risk }\end{array}$ & Unclear \\
\hline Guzman 2019, [71] & $L R$ & LR & LR & LR & $H R$ & $L R$ & UR & $\mathrm{HR}$ & LR & LR & $\mathrm{HR}$ & UR & $\begin{array}{l}\text { Moderate } \\
\text { Risk }\end{array}$ & No \\
\hline Braeckman 1997, [72] & $\mathrm{HR}$ & $H R$ & $H R$ & UC & $U C$ & $\mathrm{HR}$ & $U C$ & $\mathrm{HR}$ & $L R$ & $L R$ & $\mathrm{HR}$ & $\mathrm{HR}$ & High Risk & Unclear \\
\hline Allott 2019, [73] & $L R$ & $\mathrm{HR}$ & UR & UR & UR & UR & $L R$ & UR & LR & LR & UR & LR & $\begin{array}{l}\text { Moderate } \\
\text { Risk }\end{array}$ & Unclear \\
\hline Mills 2007, [74] & $L R$ & $L R$ & UR & UR & $L R$ & $L R$ & $L R$ & $L R$ & $L R$ & $L R$ & UR & $L R$ & Low Risk & No \\
\hline Zhang 2015, [75] & UR & $L R$ & UR & LR & UR & UR & UR & LR & $L R$ & $L R$ & UR & $L R$ & $\begin{array}{c}\text { Moderate } \\
\text { Risk }\end{array}$ & Yes \\
\hline Safwat 2018, [76] & LR & UR & UR & $L R$ & $L R$ & UR & UR & $\mathrm{HR}$ & LR & LR & UR & UR & $\begin{array}{c}\text { Moderate } \\
\text { Risk }\end{array}$ & Yes \\
\hline Ghadian 2017, [77] & UR & $\mathrm{HR}$ & UR & $L R$ & UR & UR & UR & UR & $L R$ & $L R$ & UR & UR & High Risk & Unclear \\
\hline Di Silverio 2005, [78] & LR & UR & UR & UR & UR & UR & UR & LR & $\mathrm{LR}$ & LR & $\mathrm{HR}$ & UR & $\begin{array}{l}\text { Moderate } \\
\text { Risk }\end{array}$ & Unclear \\
\hline Goodarzt 2011, [79] & $H R$ & $\mathrm{HR}$ & UR & UR & UR & UR & UR & UR & $L R$ & $L R$ & $L R$ & UR & High Risk & Unclear \\
\hline Jhang 2013, [80] & $H R$ & UR & UR & UR & UR & $\mathrm{HR}$ & UR & UR & $L R$ & $L R$ & UR & UR & High Risk & Unclear \\
\hline Page 2011, [81] & $L R$ & $L R$ & UR & UR & $L R$ & UR & UR & UR & LR & $L R$ & $L R$ & UR & $\begin{array}{c}\text { Moderate } \\
\text { Risk }\end{array}$ & Unclear \\
\hline Kacker 2014, [82] & $L R$ & UR & LR & UR & UR & $L R$ & UR & UR & LR & LR & UR & UR & $\begin{array}{c}\text { Moderate } \\
\text { Risk }\end{array}$ & Unclear \\
\hline Chung 2011, [83] & LR & $L R$ & UR & UR & UR & LR & $L R$ & $\mathrm{HR}$ & $L R$ & LR & $L R$ & $L R$ & $\begin{array}{c}\text { Moderate } \\
\text { Risk }\end{array}$ & Unclear \\
\hline Griwan 2014, [99] & $L R$ & $\mathrm{HR}$ & UR & $L R$ & UR & UR & UR & $L R$ & UR & UR & $L R$ & $L R$ & $\begin{array}{l}\text { Moderate } \\
\text { Risk }\end{array}$ & Unclear \\
\hline
\end{tabular}


A. Total Prostate Volume Changes

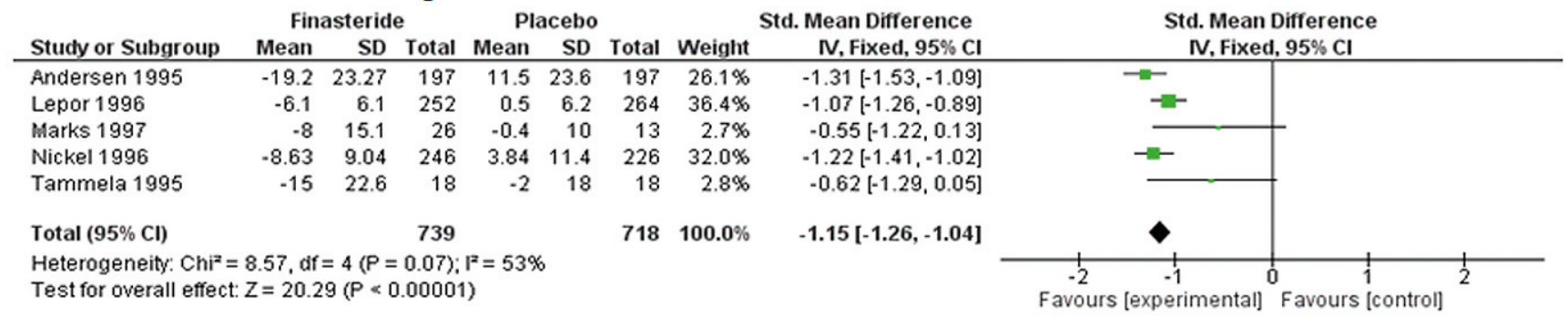

\section{B. Total Prostate Volume Changes}

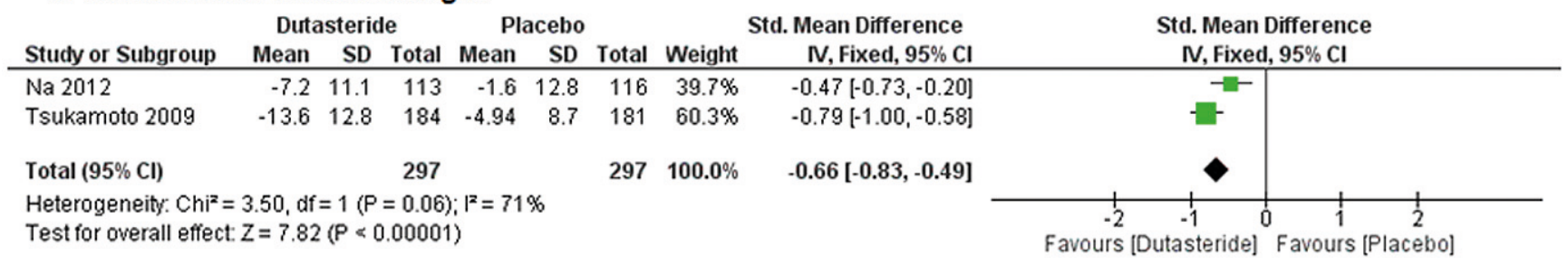

\section{Prostate Specific Antigen Changes}

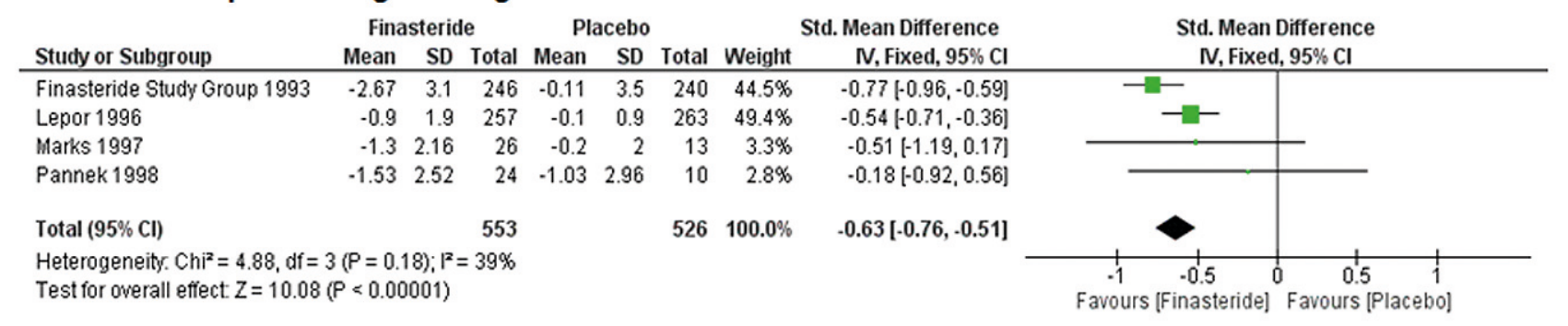

\section{Total Prostate Volume Changes at 6 months}

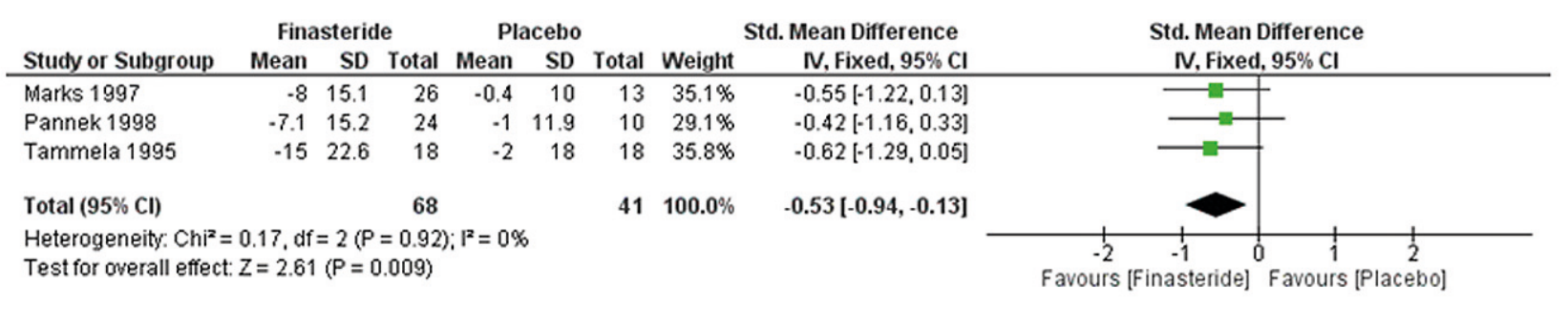

Figure 4. Meta-analysis of 5-ARI effect on prostate morphometric parameters in placebo-controlled trials. A) Forrest plot of the effect of finasteride versus placebo on total prostate volume (TPV). B) Forrest plot of the effect of dutasteride versus placebo on total prostate volume (TPV). C) Forrest plot of the effect of finasteride versus placebo on prostate-specific antigen (PSA). D) Forrest plot of the effect of finasteride on total prostate volume in placebo-controlled trials with 6 months follow-up.

$\mathrm{Cl}$-confidence interval; SD-standard deviation

teride $(-24.5 \%$ vs $-26.1 \%$ or $-9.76 \mathrm{ml}$ vs $-10.2 \mathrm{ml})$ but a significant increase of TPV $(+11.2 \%$ vs $8.66 \%)$ 12 months after discontinuation of 5-ARI therapy [52]. PSA changes were different from baseline $(-47.7 \%$ vs $49.5 \%, \mathrm{p}<0.01)$, without difference between groups $(\mathrm{p}=0.776)$. The ALFIN study reported a $-50 \%$ change $(-1.7 \pm 1.9, \mathrm{p}<0.05)$ in PSA from baseline [33]. There was no information on prostate perfusion parameters.

\section{PDE-5 inhibitors}

Yokoyama et al., randomized 612 men to receive tadalafil $2.5 \mathrm{mg}$, tadalafil $5 \mathrm{mg}$, tamsulosin $0.2 \mathrm{mg}$ or placebo for 3 months (Table 3) [20]. Authors reported non-significant changes in PSA from baseline in either tadalafil group $(-7 \%$ vs $-2 \%)$ that were similar to placebo. Pinggera et al., reported that tadalafil does not affect prostate perfusion as evaluated 


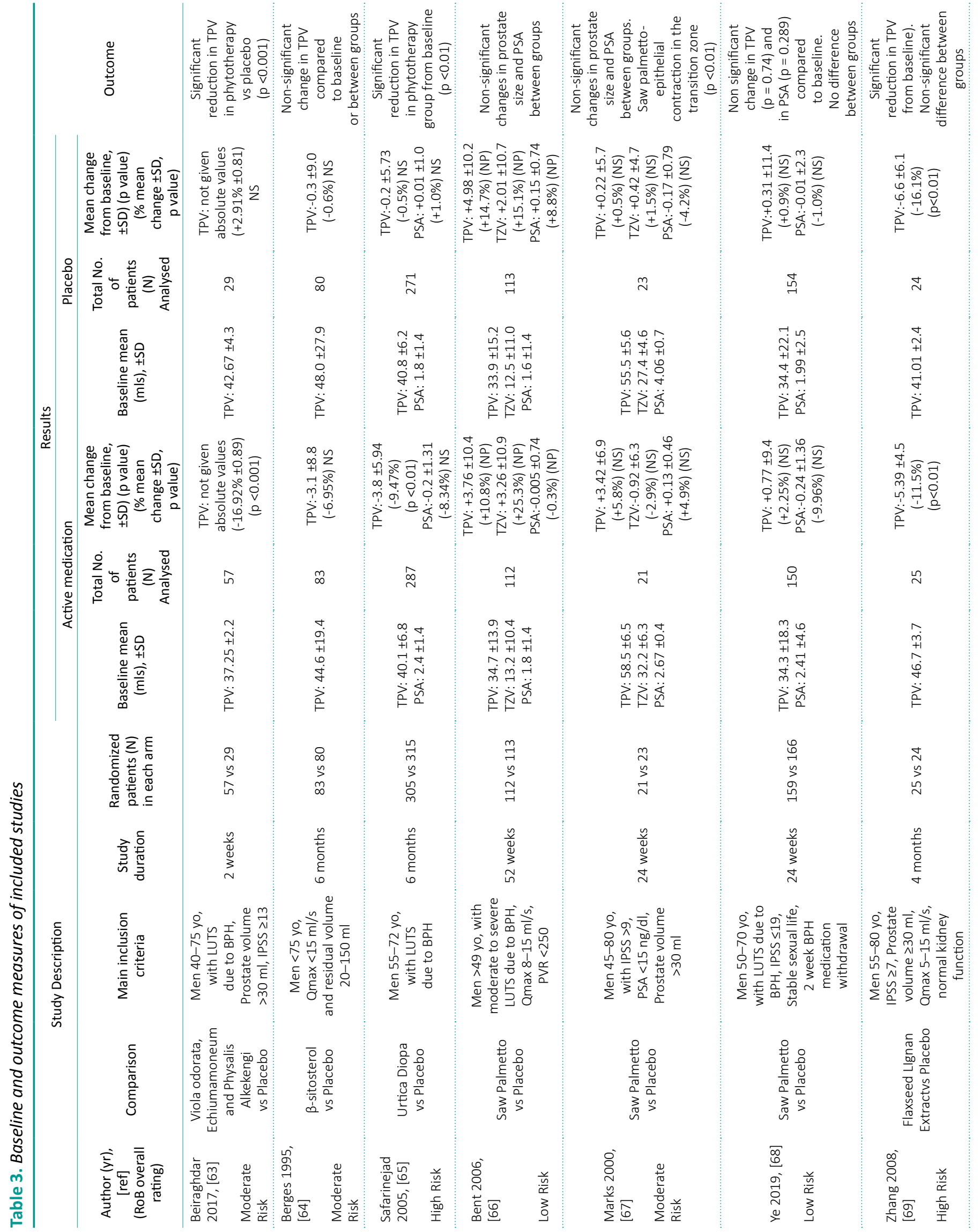




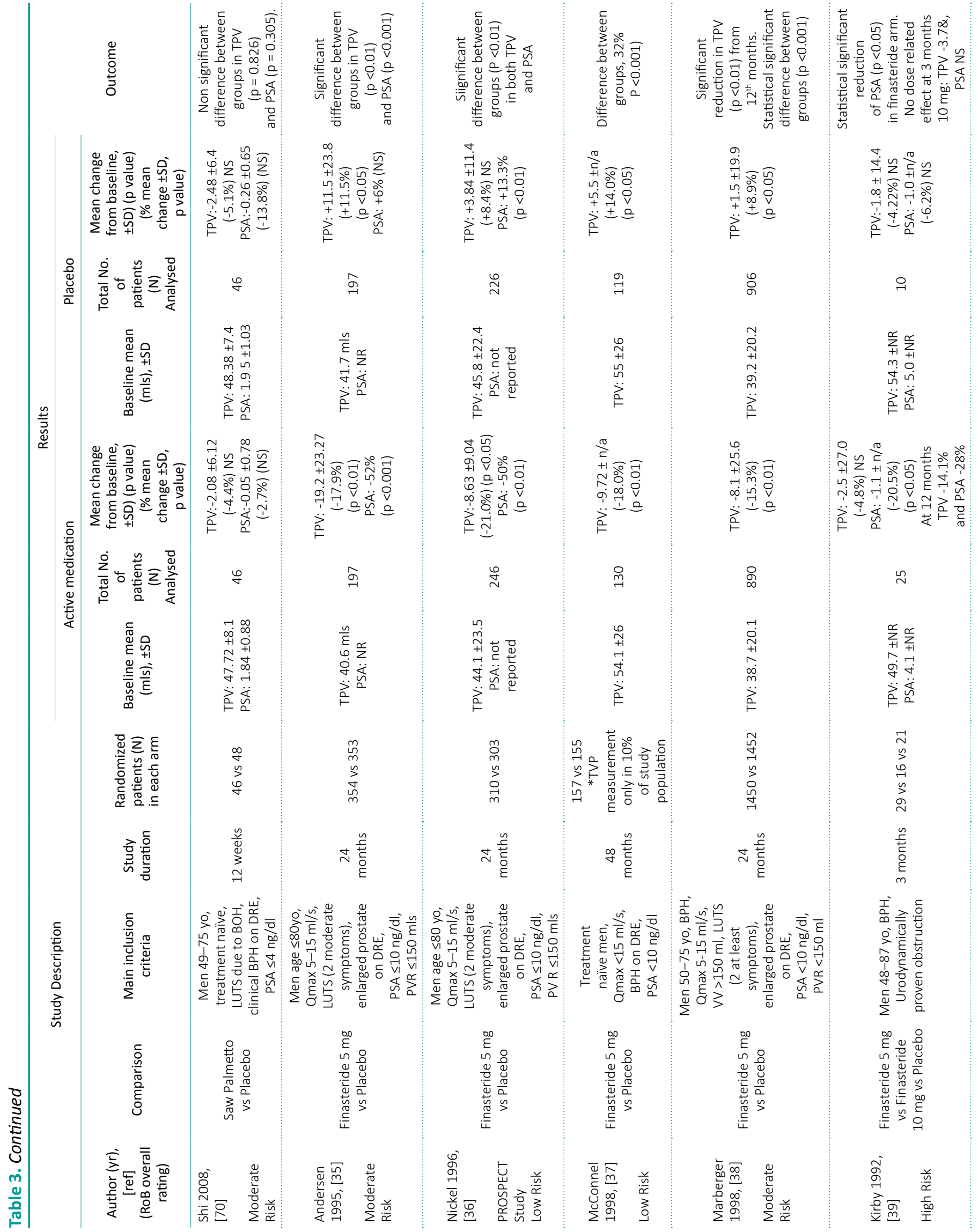




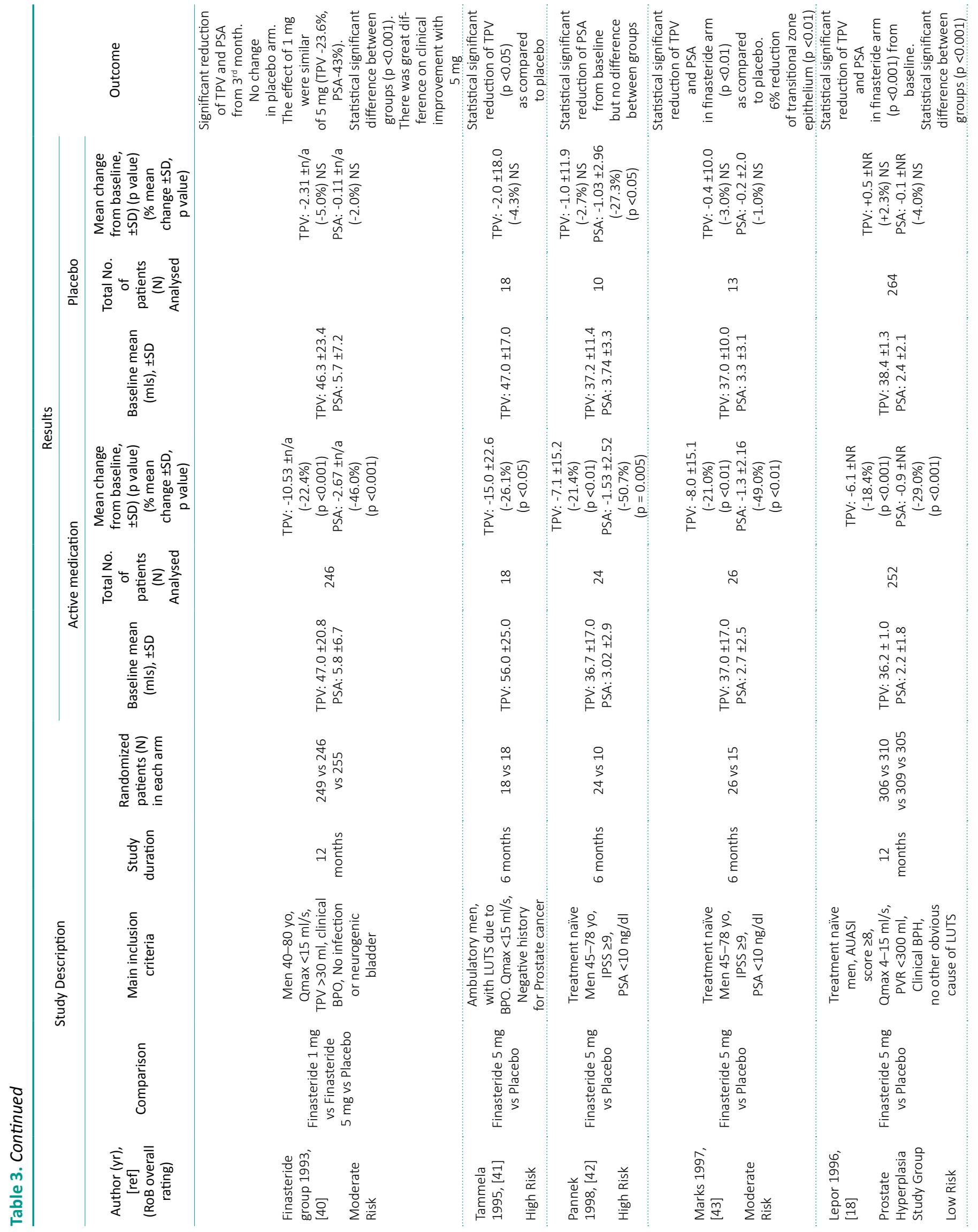




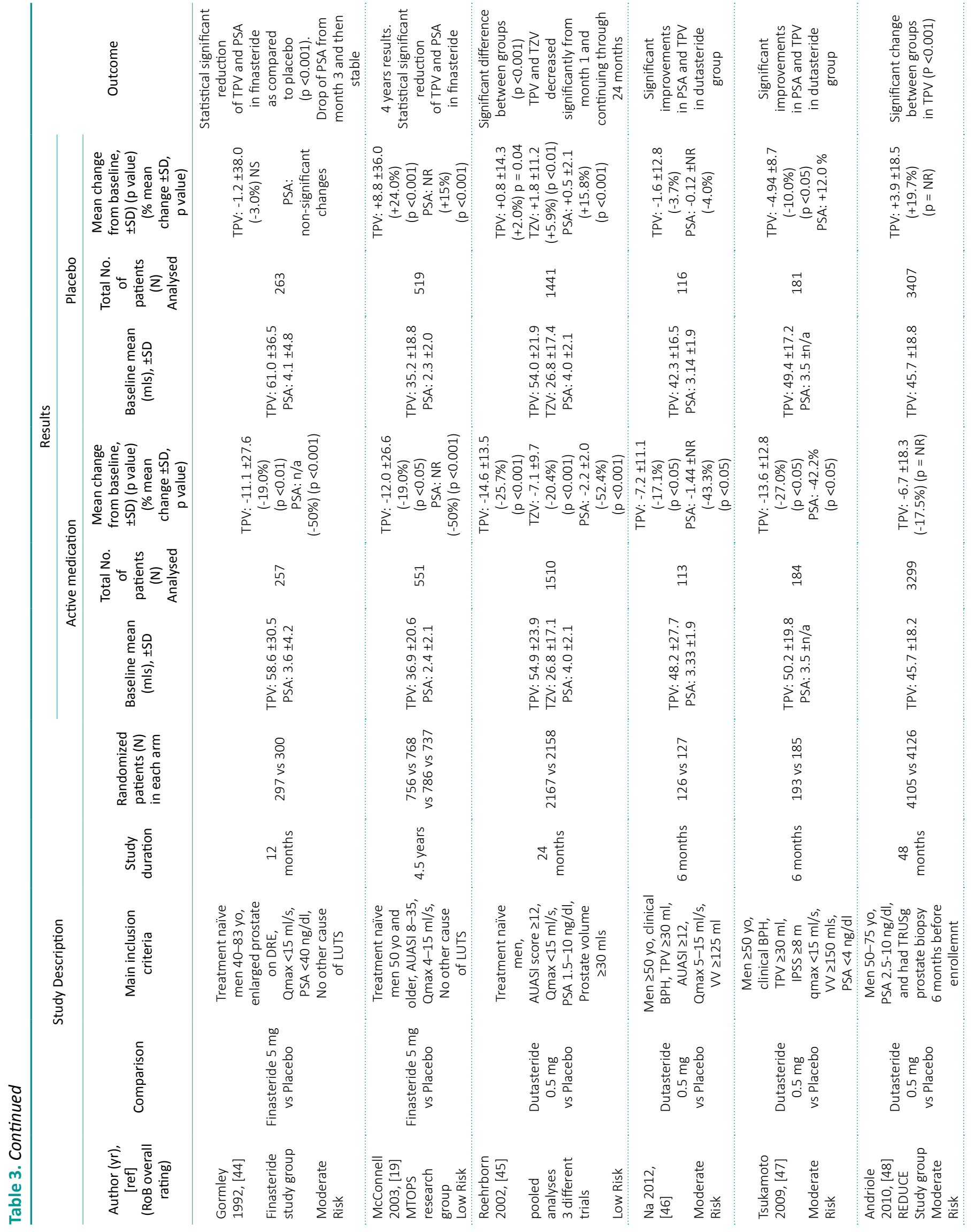




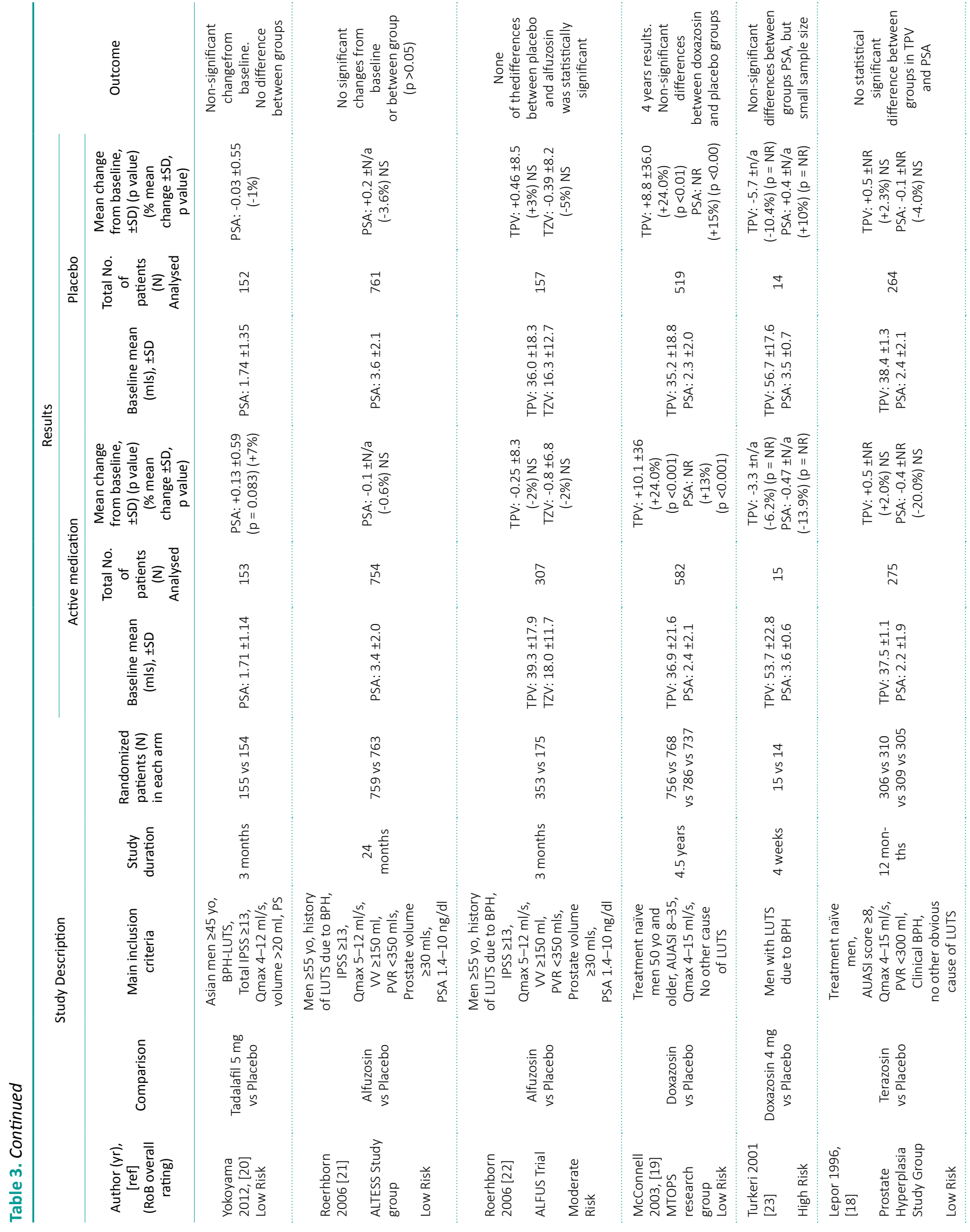




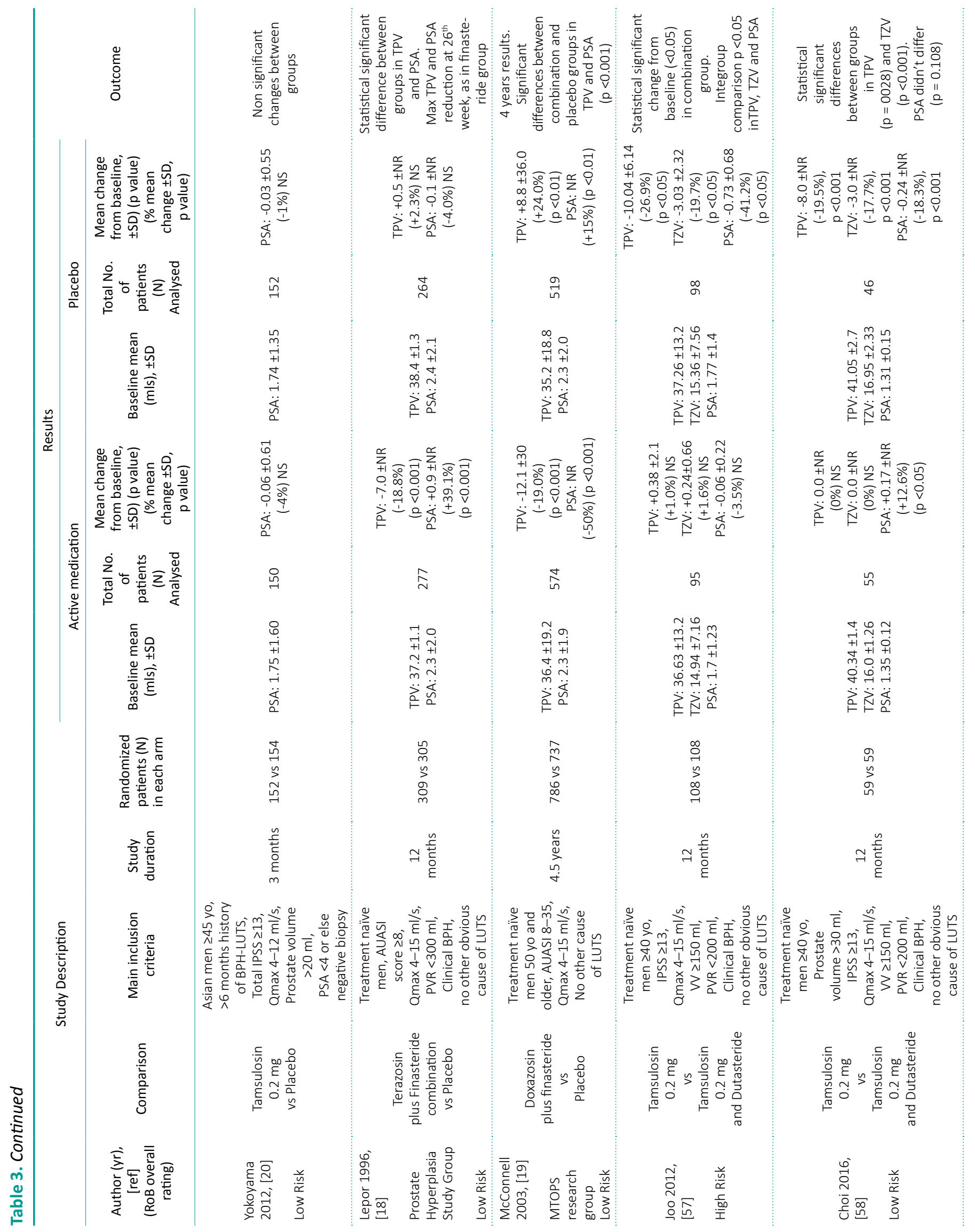




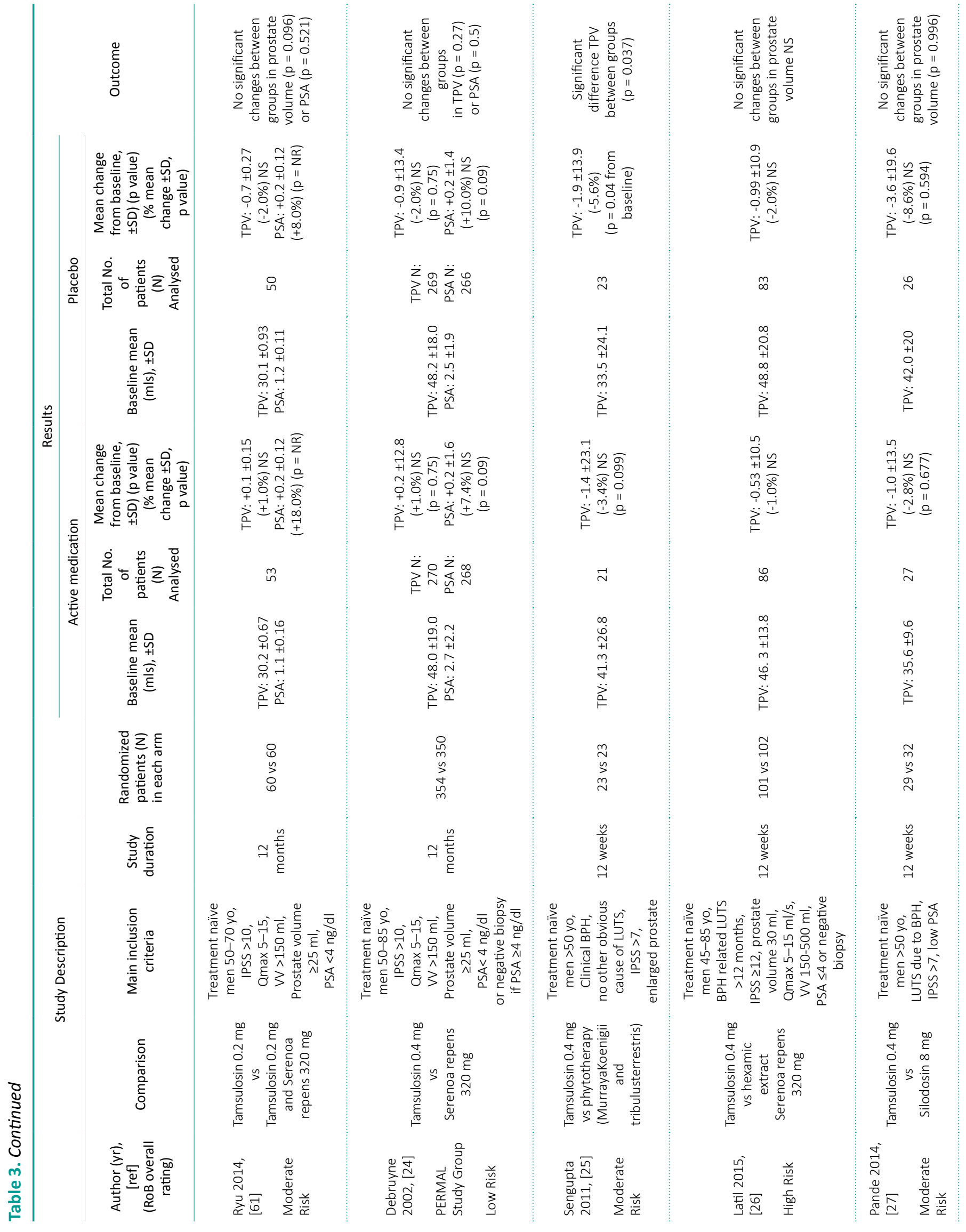




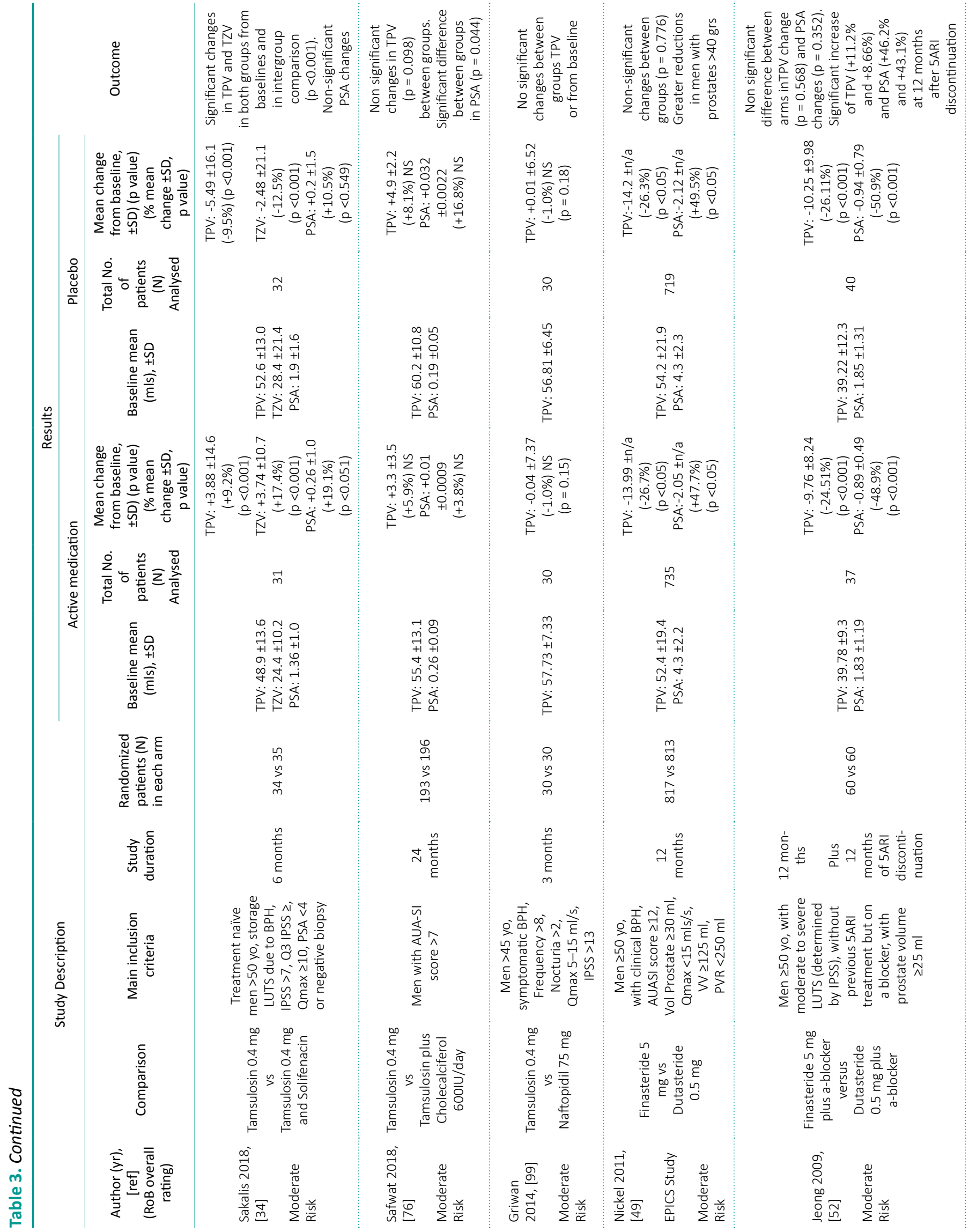




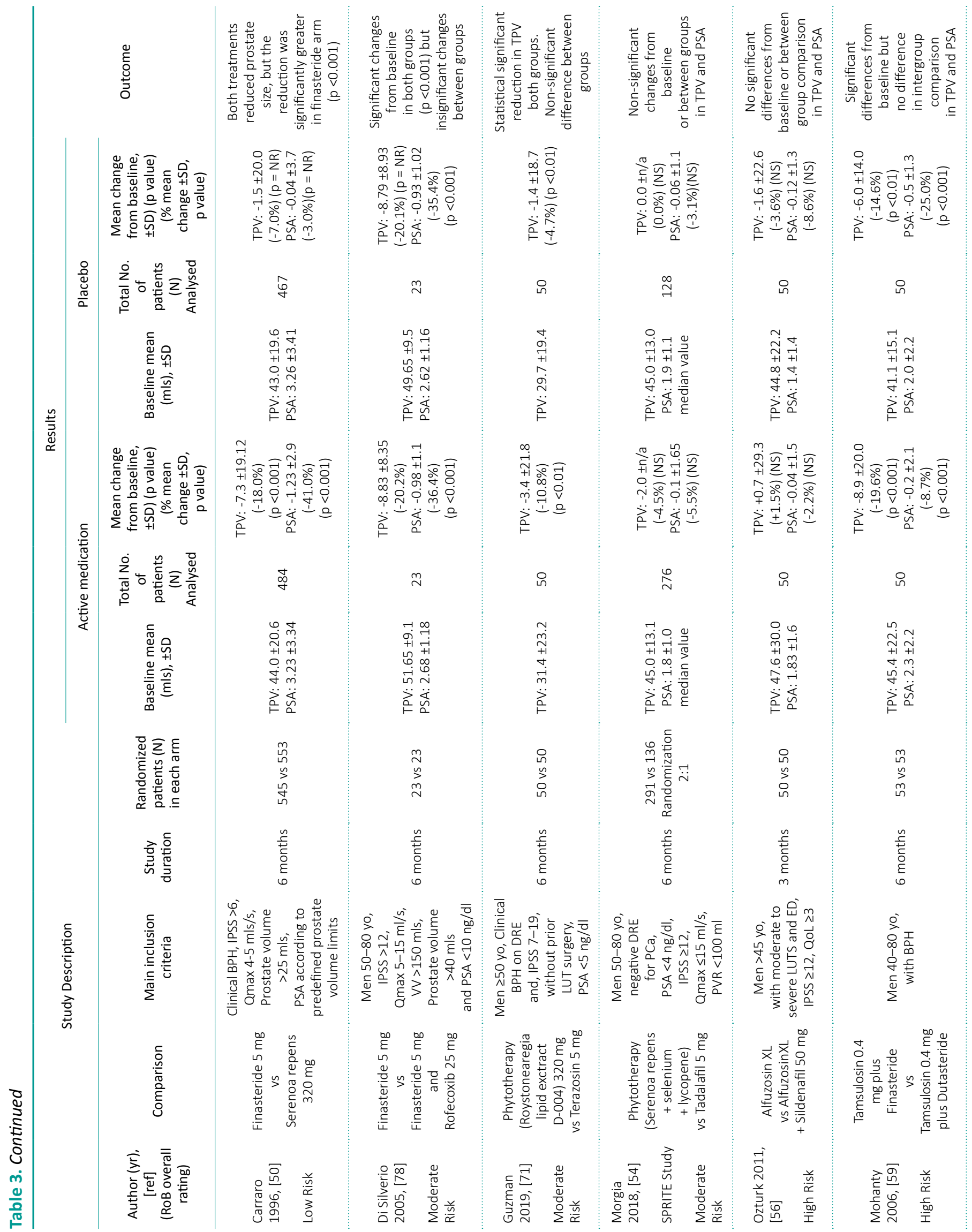




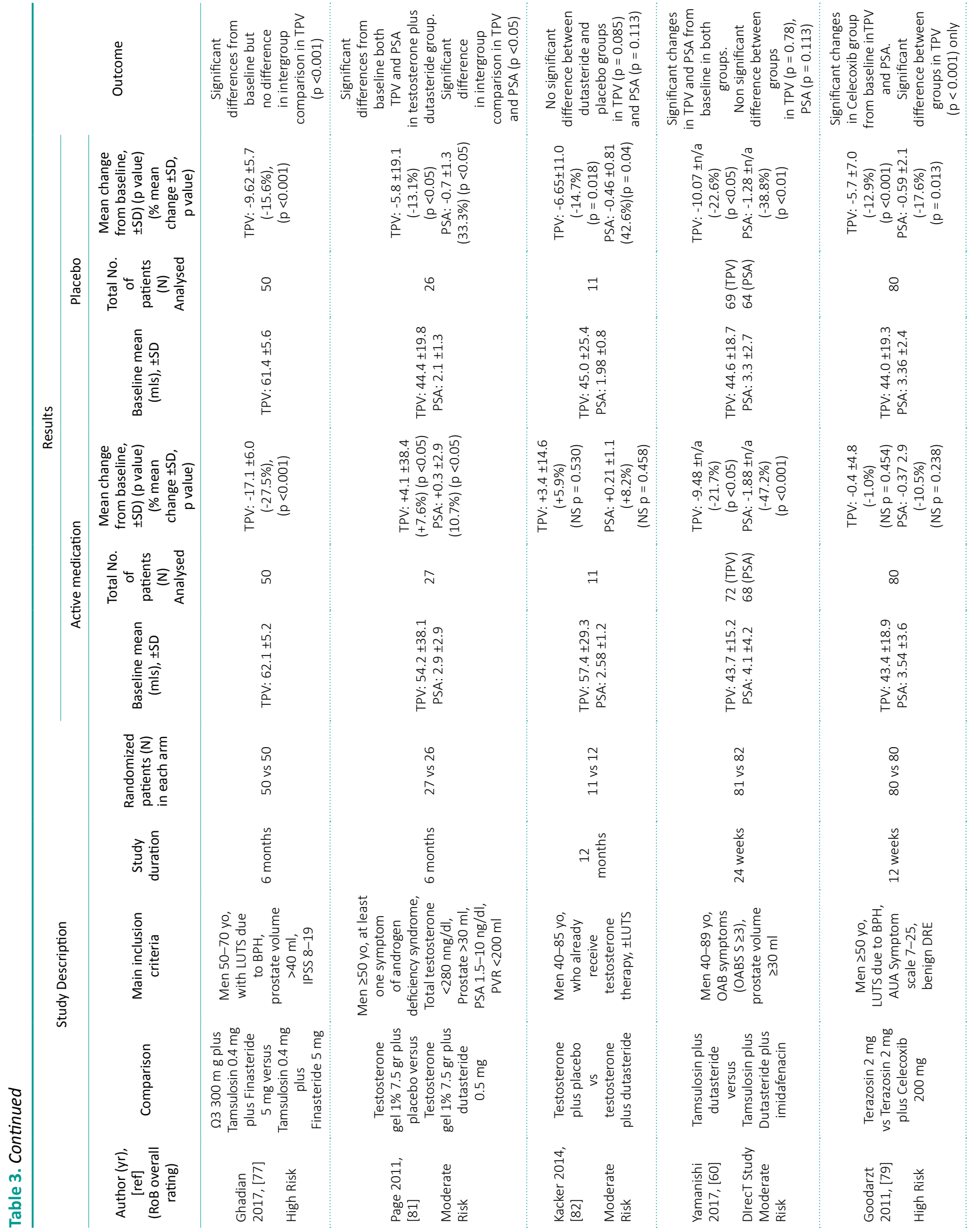




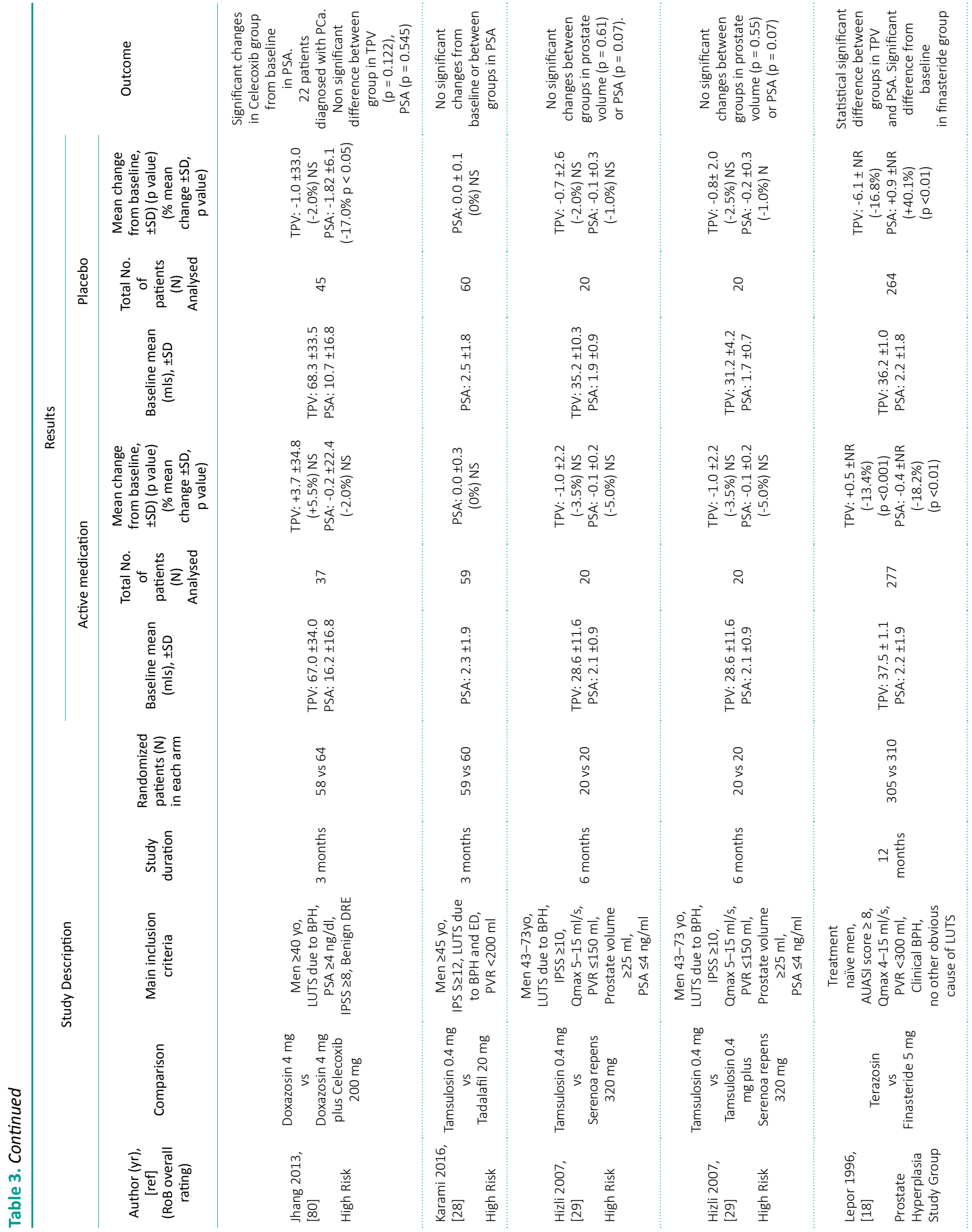




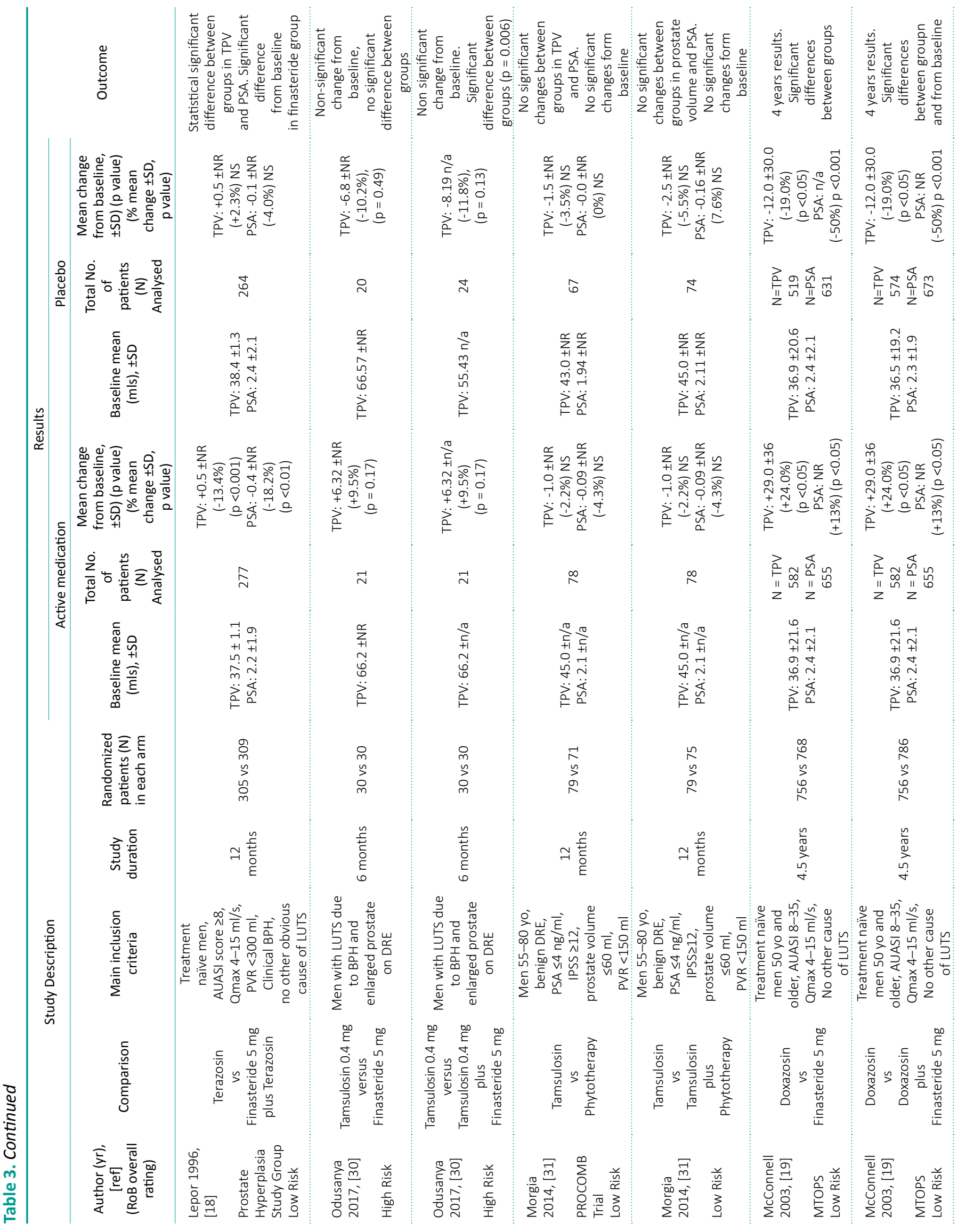




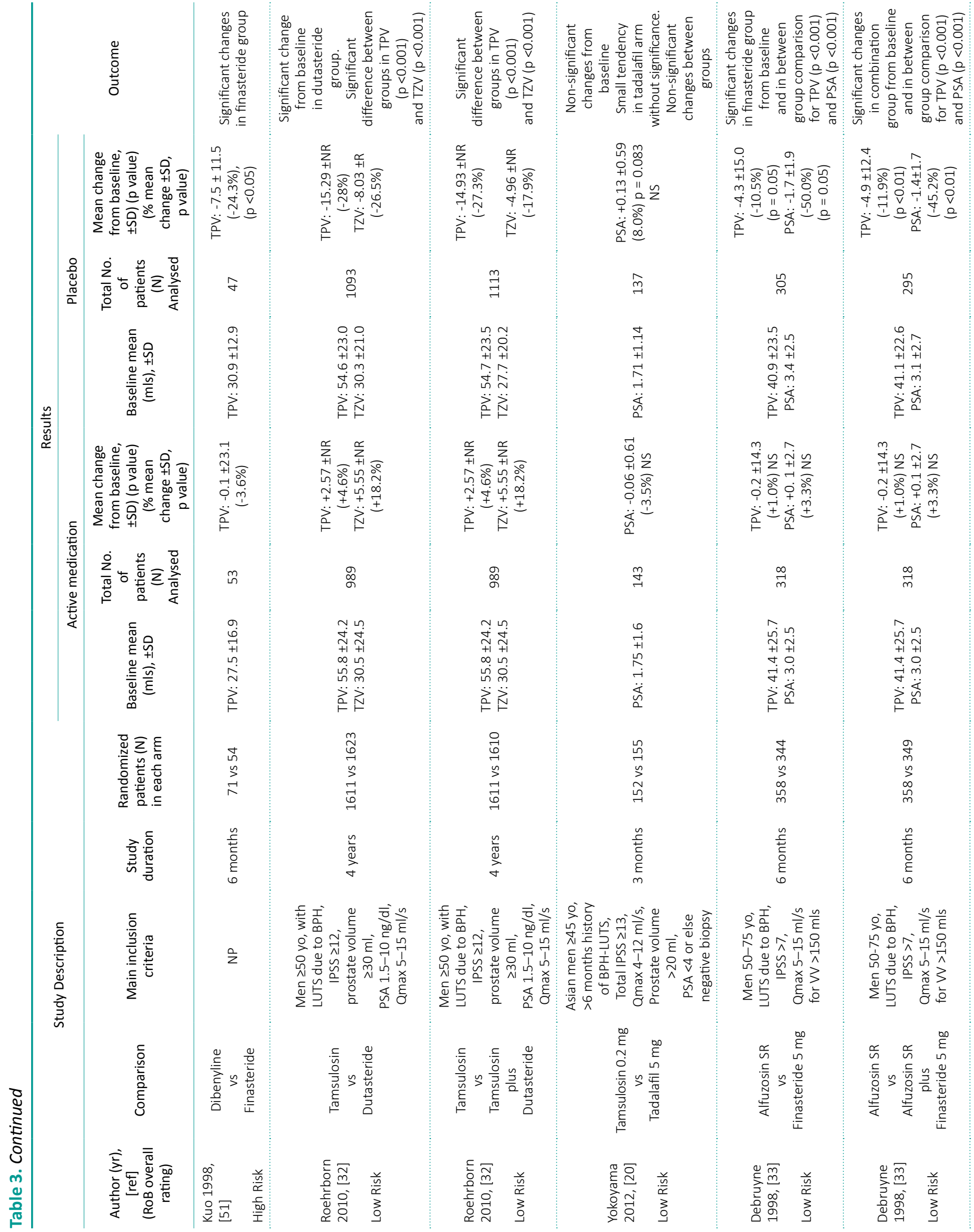




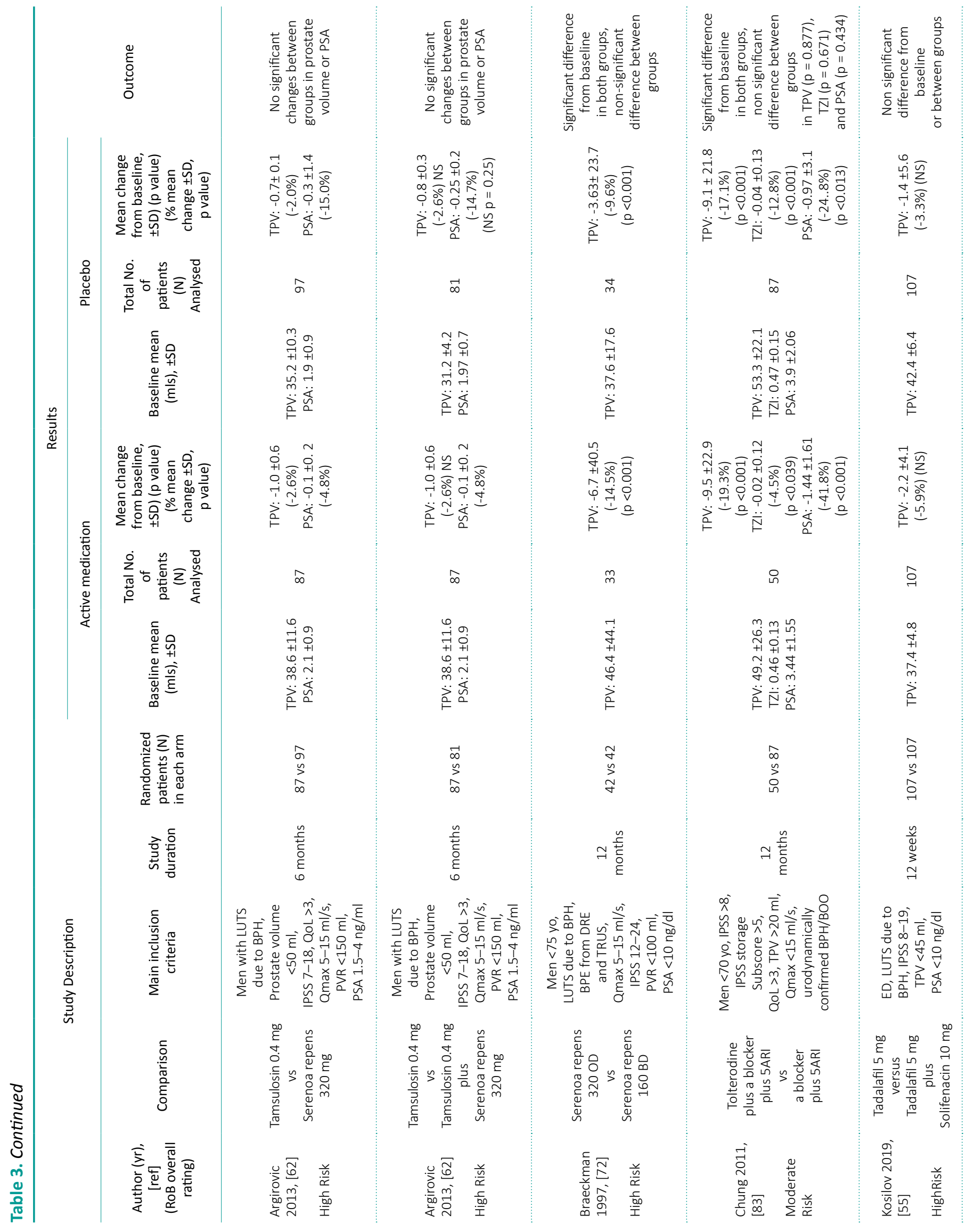




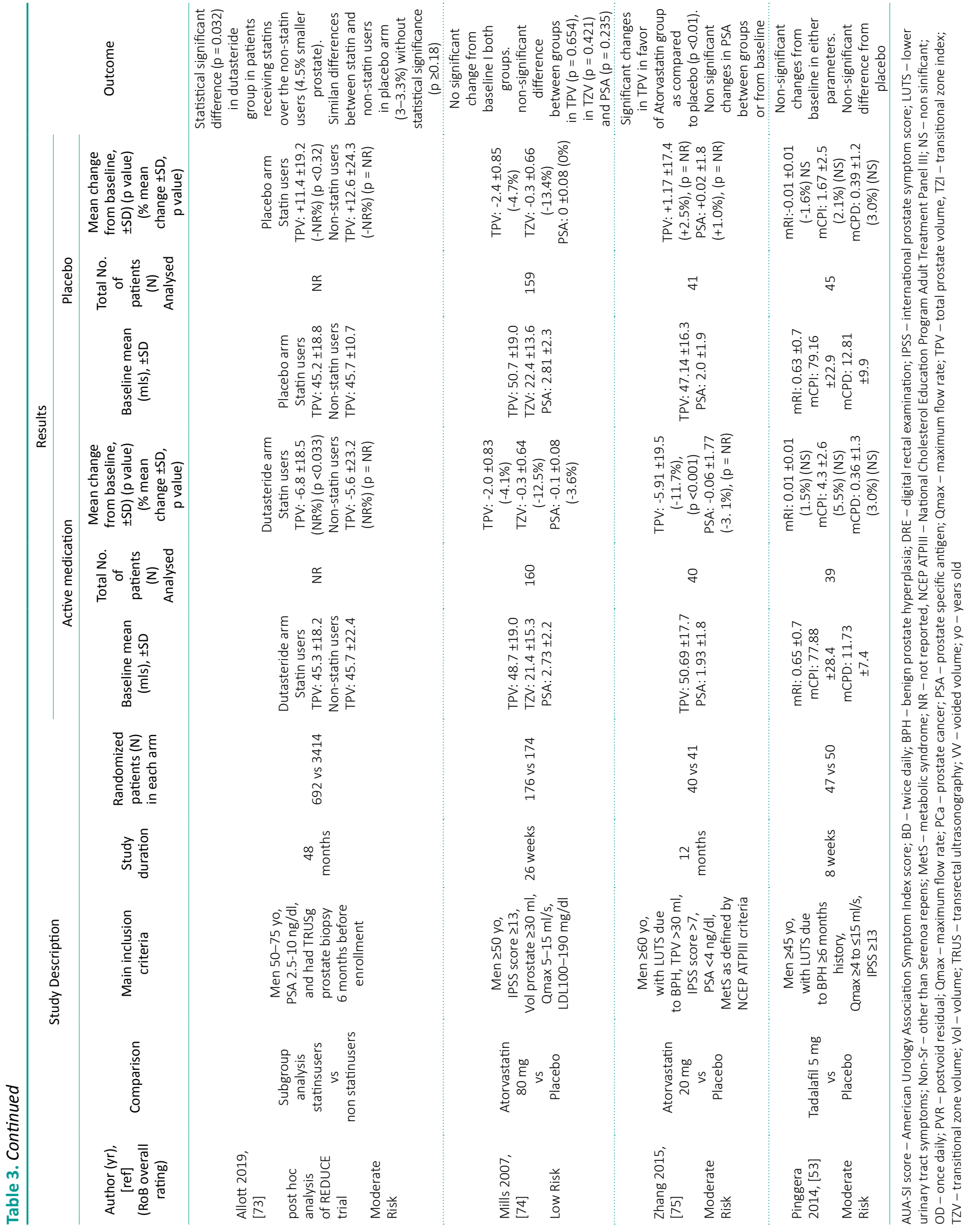


by 3 basic perfusion parameters [53]. There was no information on TPV and TZV changes.

The SPRITE study randomized men to tadalafil $5 \mathrm{mg}$ or phytotherapy [54]. No change of TPV was observed in the tadalafil arm at 6 months. Two trials with high $\mathrm{RoB}$ reported a non-significant reduction in TPV from baseline after tadalafil (-5.9\%) and sildenafil $(-3.9 \%)$ treatment $[55,56]$. PSA changes as reported in three trials were not significantly different from baseline $(-8.6 \%-0 \%)[28,54,56]$. There was no information on TZV or prostate perfusion parameters.

\section{Combination treatment}

The 12-month VA-COOP study reported a significant reduction in TPV from baseline $(-18.8 \%$ or $-7.0 \mathrm{ml}, \mathrm{p}<0.001)$ after terazosin and finasteride combination compared to non-significant changes in the placebo arm $(+2.3 \%$ or $+0.5 \mathrm{ml})$ (Table 3) [18]. MTOPS reported a similar reduction in the combination arm $(-19 \%$ or $-12.1 \mathrm{ml}$, $\mathrm{p}<0.001$ ), while TPV increased significantly in the placebo arm $(+24 \%$ or $+8.8 \mathrm{ml}, \mathrm{p}<0.01)$ [19]. MTOPS reported a 50\% PSA reduction, while VA-COOP reported an unexplained $39.1 \%$ increase from baseline. There was no information on TZV or prostate perfusion parameters.

Two 12-month studies randomized men to tamsulosin $0.2 \mathrm{mg}$ versus tamsulosin plus dutasteride combination and reported a significant reduction of TPV (-18.8\% to $-26.9 \%)$, TZV (-17.7\% to $-19.7 \%)$ and PSA $(-18.3 \%$ to $-41.2 \%)$ in combination arms and no changes in tamsulosin arms [57, 58]. Mohanty et al., compared tamsulosin plus finasteride versus tamsulosin plus dutasteride combination, and found similar TPV changes after 6 months of treatment [59].

Two 6-month RCTs assessed the influence of anticholinergics in prostate morphometric parameters in men with $\mathrm{OAB}$ and $\mathrm{BPE}[34,60]$. A moderate risk trial randomized men to tamsulosin versus tamsulosin plus solifenacin combination [34]. Authors reported a significant reduction of TPV $(-9.5 \%$ or $5.5 \mathrm{ml}, \mathrm{p}<0.001)$, TZV $(-12.5 \%$ or $-2.5 \mathrm{ml}$, $\mathrm{p}<0.001)$ and prostate perfusion $(-41 \%)$ in the combination arm. Yamahishi et al., randomized men to tamsulosin plus dutasteride alone or with imidafenacin. Both arms significantly improved TPV $(-21.7 \%$ vs $-22.6 \%)$ and PSA $(-47.2 \%$ vs $-38.8 \%)$, without significant differences between them. Three trials randomized men to tamsulosin monotherapy versus tamsulosin plus Serenoa repens (Sr). No significant differences in TPV or PSA were reported $[29,61,62]$.

\section{Phytotherapy}

Eight trials randomized men $(\mathrm{n}=1608)$ to phytotherapy versus placebo (Table 3) [63-70]. Four $\mathrm{Sr}$ trials reported non-significant changes in TPV as compared to placebo [SMD:0.12(95\%CI:-0.03 to 0.27, $\mathrm{p}=0.13$ ) (Figure 5) $[66,67,68,70]$. Non-Sr trials reported significant TPV reduction from baseline up to $-16.9 \%$ [63, 64, 69]. Two trials reported similar TZV changes to placebo at 52 and 24 weeks respectively [SMD:0.06 (95\%CI:-0.18 to $0.30, \mathrm{p}=0.64$ ) (Figure 3) [66, 67]. A small trial reported pronounced epithelial component involution in the transitional zone as compared to baseline (17.8\% to $10.7 \%$, $\mathrm{p}<0.01$ ) in the Sr group [67]. Four trials reported non-significant small effects of phytotherapy on PSA as compared to placebo [SMD: -0.06 (95\%CI: -0.21 to $0.10, \mathrm{p}=0.46$ ) (Figure 5) $[66,67,68,70]$. There was no information regarding prostate perfusion parameters.

Ten trials randomized men $(\mathrm{n}=2972)$ to phytotherapy versus active component $[24,25,26$, 29, 31, 50, 54, 62, 71, 72]. Six RCTs compared Sr to an active comparator and reported non-significant changes in TPV from baseline $(-7 \%$ to $-2 \%$ or $-2.0 \mathrm{ml}$ to $-0.7 \mathrm{ml})[24,26,29,31,50,62]$. A single trial reported significant TPV change from baseline after $\mathrm{Sr} 320 \mathrm{mg}$ once daily (-14.5\%, $\mathrm{p}<0.001)$ or Sr $160 \mathrm{mg}$ twice daily for 12 months $(-9.6 \%, \mathrm{p}<0.001)$ [72]. Two non-Sr trials reported significant reduction in TPV as compared to baseline $(-5.6 \%$ and $-10.8 \%)$ [25, 71]. Six trials reported non-significant changes in PSA as compared to baseline or to comparator $(-15 \%$ to $+10 \%$ or $-0.3 \mathrm{ng} / \mathrm{dl}$ to $+0.2 \mathrm{ng} / \mathrm{dl}$ ) $[24,29,31,50,62]$. There was no information regarding TZV and prostate perfusion parameters.

\section{Other medications}

A post hoc analysis of the REDUCE trial classified men on dutasteride as statin and non-statin users (Table 1) [73, 48]. Authors reported a significant TPV change from baseline $(-15.8 \%$ or $-6.8 \mathrm{ml}$, $\mathrm{p}=0.033)$ in the statin users' subgroup as compared to the non-statin users. The effect of dutasteride on lowering TPV was roughly 10 -fold greater than the statin-associated effect at year 2 (p <0.001) and year $4(\mathrm{p}<0.001)$ [73]. A 26-week RCT compared atorvastatin $80 \mathrm{mg}$ versus placebo and reported no difference from baseline or between groups in TPV $(-4.1 \%$ vs $-4.7 \%, \mathrm{p}=0.654)$, in TZV $(-12.5 \%$ vs $-13.4 \%, \mathrm{p}=0.421)$ and in PSA $(-3.6 \%$ vs $0 \%, \mathrm{p}=0.235$ ) [74]. In contrast, a 12-month trial reported a significant difference in TPV in favor 


\begin{tabular}{|c|c|c|c|c|c|c|c|c|c|}
\hline \multicolumn{10}{|c|}{ A. Total Prostate Volume Changes SrR versus Placebo } \\
\hline \multirow[b]{2}{*}{ Study or Subgroup } & \multicolumn{3}{|c|}{ Serenoa repens } & \multicolumn{3}{|c|}{ Placebo } & \multicolumn{2}{|c|}{ Std. Mean Difference } & \multirow{2}{*}{$\begin{array}{l}\text { Std. Mean Difference } \\
\text { IV, Fixed, } 95 \% \mathrm{Cl}\end{array}$} \\
\hline & Mean & SD & Total & Mean & SD & Total & Weight & IV, Fixed, $95 \% \mathrm{Cl}$ & \\
\hline Ye 2019 & 0.77 & 9.4 & 150 & 0.31 & 11.4 & 154 & $45.9 \%$ & $0.04[-0.18,0.27]$ & \\
\hline Shi 2008 & -2.08 & 6.12 & 46 & -2.48 & 6.4 & 46 & $13.9 \%$ & $0.06[-0.35,0.47]$ & \\
\hline Bent 2006 & 3.76 & 10.4 & 112 & 1.98 & 10.2 & 113 & $33.8 \%$ & $0.17[-0.09,0.43]$ & 一늠는 \\
\hline Marks 2000 & 3.42 & 6.9 & 21 & 0.22 & 5.7 & 23 & $6.4 \%$ & $0.50[-0.10,1.10]$ & \\
\hline Total $(95 \% \mathrm{Cl})$ & & & 329 & & & 336 & $100.0 \%$ & $0.12[-0.03,0.27]$ & \\
\hline $\begin{array}{l}\text { Heterogeneity: } \mathrm{Chi}^{2}= \\
\text { Test for overall effect }\end{array}$ & $\begin{array}{l}2.19, \mathrm{df}= \\
Z=1.53\end{array}$ & $\begin{array}{l}=3(P= \\
(P=0 .\end{array}$ & $\begin{array}{l}=0.53): 1 \\
13)\end{array}$ & $I^{2}=0 \%$ & & & & & $\begin{array}{cccc} & 1 & 1 & + \\
-1 & -0.5 & 0 & 0.5 \\
\text { Favours [Serenoa repens] } & \text { Favours [Placebo] }\end{array}$ \\
\hline
\end{tabular}

B. Transitional Zone Volume Changes SrR versus Placebo

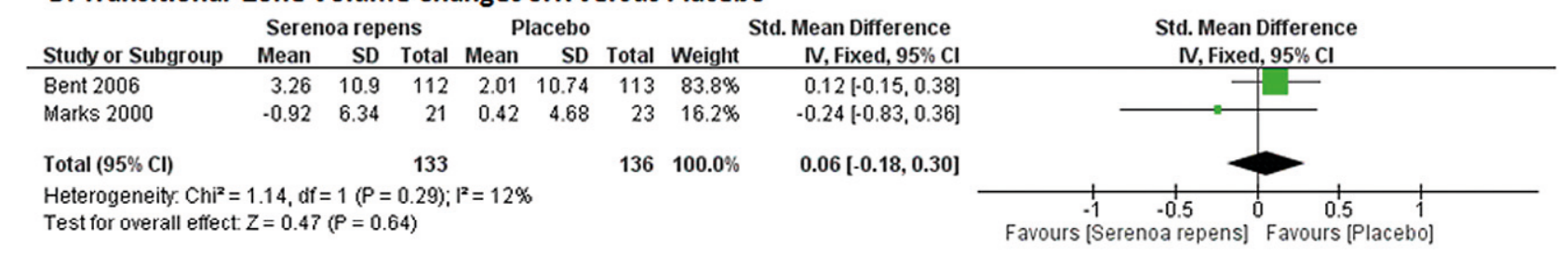

\section{Prostate Specific Antigen Changes SrR versus Placebo}

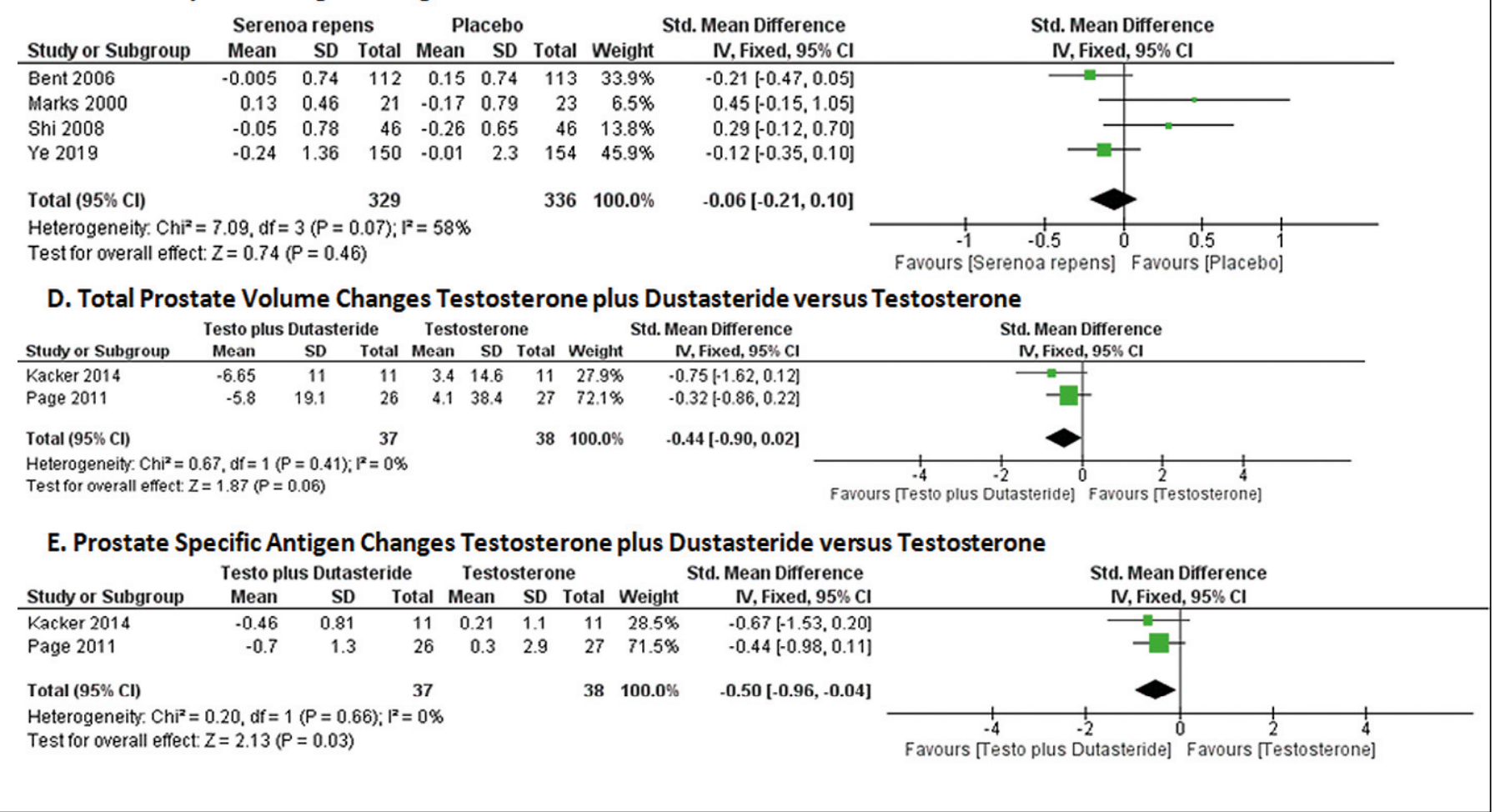

Figure 5. Meta-analysis of Serenoa repens (Sr) and testosterone effect on prostate morphometric parameters effect in placebo-controlled trials. A) Forrest plot of the effect of Sr versus placebo on total prostate volume (TPV). B) Forrest plot of the effect of Sr versus placebo on transitional zone volume (TZV). C) Forrest plot of the effect of Sr versus placebo on prostatespecific antigen (PSA). D) Forrest plot of the effect of testosterone plus dutasteride combination versus testosterone monotherapy on total prostate volume (TPV). E) Forrest plot of the effect of testosterone plus dutasteride combination versus testosterone monotherapy on PSA.

$\mathrm{Cl}$ - confidence interval; SD - standard deviation

of atorvastatin group as compared to placebo $(-11.7 \%$ vs $+2.5 \%, \mathrm{p}<0.01)$, changes which were more pronounced in obese patients compared to normoweight individuals [75].
A single study compared cholecalciferol 600 IU plus tamsulosin versus tamsulosin monotherapy and reported non-significant TPV changes between groups $(+8.1 \%$ vs $+5.9 \%, \mathrm{p}=0.098)$ at 24 months [76]. 
S3-fatty acids in combination with tamsulosin and finasteride were non-superior to tamsulosin plus finasteride combination in reducing TPV [77]. Di Silverio studied the effect of rofecoxib $25 \mathrm{mg}$ with finasteride versus finasteride monotherapy and reported comparable reductions in TPV $(-20.1 \%$ vs $-20.2 \%)$ and PSA (-35.4\% vs $-36.4 \%)$ at 6 months [78]. They reported an accelerated effect in rofecoxib group. The effect of celecoxib was tested in two studies against terazosin and doxazosin [79, 80]. The first reported that celecoxib reduces significantly TPV $(-12.9 \%)$ and PSA $(-17.6 \%)$ and the latter reported a significant PSA change $(-17.0 \%)$ only. Two RCTs studied the effect of testosterone replacement in TPV and PSA of men with androgen deficiency syndrome [81, 82]. Both studies reported an increase in TPV $(+7.6 \%$ and $+5.9 \%)$ and PSA $(10.7 \%$ and $+8.2 \%)$ after testosterone supplementation. The coadministration of dutasteride spares prostate from androgenic stimulation since both TPV (-13.1\% and $-14.7 \%)$ and PSA (-33.3\% and $-42.6 \%)$ were reduced significantly. The treatment effect was considered moderate in favor of combination regarding TPV [SMD: -0.44 (95\%CI: -0.90 to $0.02, \mathrm{p}=0.06$ ) (Figure 3) and PSA change [SMD: -0.50 (95\%CI: -0.96 to $0.04, \mathrm{p}=0.03$ ).

\section{Placebo arm}

In phytotherapy trials, the changes in TPV were from $-5.1 \%$ to $+2.91 \%$ and in PSA from $-4.2 \%$ to $-1.0 \%$ [63-70]. A trial with 12-month follow-up reported $+14.7 \%$ increase in TPV and $+8.8 \%$ increase in PSA [66]. In short-term RCTs with $\alpha-1$ blockers, the changes in TPV and PSA were not significant, ranging from $2.3 \%$ to $3 \%$ and $-4 \%$ to $+10 \%$ respectively. In 5 -ARI trials TPV change was reported between $-10.0 \%$ and $-2.7 \%$ in 6 -month studies, $-5.0 \%$ to $-2.3 \%$ in 12 -month studies, $+2.0 \%$ to $+14.0 \%$ in $24-$ month studies [18, 19, 35-48]. REDUCE and MTOPS trials, both with long follow-up, reported TPV change $+19.7 \%$ and $+24.0 \%$ respectively $[48,19]$. The changes in PSA were $-6.0 \%$ to $-1.0 \%,-5.0 \%$ to $-2.0 \%$ and $+6 \%$ to $+15.8 \%$ respectively.

\section{DISCUSSION}

Herein, we systematically reviewed the effect of pharmacotherapy on prostate morphometric parameters, namely TPV, TZV, PSA and prostate perfusion. The strengths of this review include the systematic and transparent approach to analyze the evidence base, including the Cochrane review methodology, the adherence to PRISMA guidelines and a-priori written protocol. We also used a comprehensive approach to determine RoB and to include studies with well-defined protocol assessing morphometric parameters.

The weaknesses relate to the limitations of the body of evidence that we analyzed. Based on AHRQ standards, 16 RCTs were considered as low-risk, 31 RCT as moderate-risk and 20 as high-risk. Thirteen out of 28 placebo-controlled trials were considered of moderate-risk. Most trials were powered to assess post-treatment changes in clinical parameters such as relevant questionnaires or flow test. Only $10 \mathrm{RCTs}$ were powered to assess changes in morphometric parameters as a primary outcome. An additional methodological issue relates to the technique used to evaluate prostate parameters. To overcome measurement bias, we included studies that describe in detail the method of volume calculation. Concerning PSA and perfusion parameters, we relied on data provided by each group.

$\alpha$-blockers do not affect TPV, TZV or PSA. Studies with long-term follow-up report changes similar to placebo, while the observed significant differences from baseline result from physiologic growth. Animal experiments demonstrated that sympathomimetics induce prostate hyperplasia, whereas quinazoline-based $\alpha$-blockers exert apoptotic effect on human prostate cancer cell cultures [8]. This in vitro effect is not evident in clinical setting [83]. There is evidence that tamsulosin improves prostate perfusion, possibly by the antagonistic action on $\alpha 1 \mathrm{~A}$ - and $\alpha 1 \mathrm{D}$-adrenoceptors of vesical arteries $[12,84]$. A single RCT in OAB population reported increased perfusion up to $+149 \%$, which was similar to previous findings $(+132.8 \%)$, hence the beneficial effect of tamsulosin on LUTS [85].

Robust evidence supports the effect of 5-ARIs on TPV, TZV and PSA. Dihydrotestosterone (DHT) induces prostate growth via enhanced proteinosynthesis and reduced apoptotic rates [86]. 5-ARIs reduce TPV, TZV and PSA in at least $85.3 \%$ of patients after six to twelve months of treatment [87]. A headto-head comparison of finasteride and dutasteride showed similar efficacy, but dutasteride effect appears sooner [49, 52]. A pooled analysis of dutasteride trials reports significant changes of TPV starting at $1^{\text {st }}$ month of treatment, as a result of the faster DHT suppression $[45,47]$. These changes reach the maximum effect at 12 months and this change is sustained thereafter [18, 19, 35-38, 40, 44]. DHT increases prostatic blood flow via increased expression of VEGF [86]. Finasteride downregulates VEGF and reduces prostate blood flow as early as 7 days after administration $[11,86]$. Preliminary literature search on single-arm studies revealed two dutasteride 
single-arm trials reporting a reduction in perfusion parameters [88, 89].

Even though there is little evidence, PDE5 inhibitors do not affect TPV, TZV and PSA. Studies on human prostatic tissue strips, suggested that upregulation of intracellular cGMP by PDE5 inhibition decreases smooth muscle tone and might attenuate prostate cells proliferation [3, 10, 90, 91]. Animal models of chronic pelvic ischemia demonstrated that PDE5 inhibitors increase cGMP levels and improve lower urinary tract perfusion [10]. Using contrastenhanced ultrasound, an observational study demonstrated improvements in prostate perfusion after tadalafil administration [10, 92]. In men at high-risk for endothelial dysfunction, tadalafil significantly improves flow-mediated dilation of brachial artery as compared to controls $[21,93]$. However, these vasoactive effects of PDE5 inhibitors were not evident at clinical level [9, 10, 92, 94, 95]. A single RCT did not report any significant effect on prostate perfusion parameters [53].

The overall effect of phytotherapy on prostate morphometric parameters is ambiguous. Both placebocontrolled and active medication-controlled trials on $\mathrm{Sr}$ reported no significant difference from comparators, while non-Sr trial reported a significant reduction in TPV. These trials are characterized by high heterogeneity and poor quality. Conclusions from phytotherapy trials are difficult due to differences in consistency, concentration or extraction techniques. As a result, the biological activity might differ even among studies with same extracts. A recent meta-analysis reported significant reduction in TPV and non-significant increase of PSA after administration of hexanic extract of Sr [96].

Combination treatment is indicated when monotherapy fails to control symptoms. According to European Association of Urology (EAU) guidelines, $\alpha$-blockers are combined with 5-ARIs to improve residual voiding LUTS or with an anticholinergic for residual storage symptoms [1]. CombAT reported similar TPV changes between combination and dutasteride monotherapy arm (-27.3\% vs $-28.0 \%)$ [32]. TZV changes differ, almost statistically significantly $(-17.9 \%$ vs $-26.5 \%, p=0.052)$. In the case of $\alpha$-blocker with anticholinergic combination the data is limited. A single RCT reported significant reduction in TPV, TZV and perfusion parameters with combination of solifenacin and tamsulosin as opposed to tamsulosin monotherapy [34]. There is no data on the effect of $\beta 3$-agonists on TPV. Evidence from basic science shows that mirabegron improves bladder wall blood flow and bladder dysfunction through amelioration of pelvic blood flow [97].
Statins reduce TPV, albeit ten times less than dutasteride [73]. Recent evidence shows that atorvastatin has pro-cell apoptotic action, a pro-cellular adhesion effect, a pro-proliferation effect and an antiinflammatory action via reduction of Interleukin-6 and IGF-1 [75]. Cholecalciferol and rofecoxib did not differ from their comparators. By contrast, celecoxib reduces both TPV and PSA [79, 80]. Testosterone replacement therapy restores DHT levels, thus TPV and PSA do not change further from a saturation point [82]. Dutasteride reduces TPV, PSA in men who receive testosterone replacement therapy, an effect that validates the influence of intraprostatic DHT on morphometric parameters.

A single summary for the effect of medications on prostate morphometric parameters is not possible. The degree of heterogeneity renders inappropriate any formal data pooling. The reasons of heterogeneity were the differences in study design, in follow-up duration, in sample size, in drop-out rates, in the inadequacy of reporting standards and in the forced unilateral regression to the mean (due to inclusion/ exclusion criteria other than volume such as uroflowmetry). In addition, a small number of trials were powered enough to detect changes in morphometric parameters while others were characterized as lowquality due to high risk of bias [100]. The placebo response differs surprisingly among trials. A similar effect has been previously described [98]. The relevant mechanisms of this effect are poorly understood.

\section{CONCLUSIONS}

A detailed review of the effect of medical therapy on prostate morphometric parameters has been presented. The 5-ARIs show large effect size in reducing TPV as compared to placebo. There is no difference between finasteride and dutasteride but data support an earlier influence of dutasteride on TPV. Quinazolin-based $\alpha$-blockers are associated with significant TPV changes in 4-year trials which are similar to placebo and represent the natural growth of prostate. Non-Sr phytotherapy appears to reduce TPV in contrast to a non-effect of Sr, but relevant studies suffer from moderate or high risk of bias. PDE5-inhibitors' trials reported non-significant TPV changes. Among other medications, atorvastatin and celecoxib were found to significantly reduce TPV. A large effect on TZV is observed after either 5-ARI monotherapy or after combination treatment with an $\alpha$-blocker, but the reduction in the latter group is less. PSA changes are significant in patients receiving 5ARI monotherapy or in combination. No other treatment class appears to affect PSA. There is less robust evidence to suggest that tamsulosin improves prostate 
perfusion while tadalafil has no effect on clinical perfusion parameters.

\section{CONFLICT OF INTEREST}

The authors report no conflicts of interest.

\section{DEPARTMENT AND INSTITUTION WHERE THE WORK WAS CARRIED OUT:}

Agios Pavlos General Hospital of Thessaloniki, Greece $2^{\text {nd }}$ Department of Urology, Aristotele University of Thessaloniki, Greece

\section{References}

1. Gravas S, Cornu JN, Gacci M, Gratzke C, Herrmann TRW, Mamoulakis $C$ et al. EAU guidelines on management of non-neurogenic male lower urinary tract symptoms (LUTS), incl. benign prostatic obstruction (BPO). Edn. Presented at the EAU Annual Congress Barcelona. Arnhem: EAU Guideline Office.

2. Abrams P, Cardozo L, Fall M, et al. the standardization of terminology in lower urinary tract function: report from the standardization sub-committee of the International Continence Society. Neurourol Urodyn. 2002; 61: 37-49.

3. Jacobsen SJ, Girman CJ, Lieber MM. Natural history of benign prostatic hyperplasia. Urology. 2001; 58: 5-16.

4. Crawford ED, Wolson SS, McConnell JD, et al for the MTOPS Research group. Baseline factors as predictors of clinical progression of benign prostatic hyperplasia in men treated with placebo. J Urol. 2006; 175: 1422-1427.

5. Kozminski MA, Wei JT, Nelson J, Kent DM Baseline characteristics predict risk of progression and response to combined medical therapy for benign prostatic hyperplasia (BPH). BJU Int. 2015; 115: 308-318.

6. Loeb S, Kettermann A, Carter HB, Ferrucci L, Metter EJ, Walsh PC. Does prostate growth confound prostate specific antigen velocity? Data from the Baltimore Longitudinal Study of Aging. J Urol. 2008; 180: 1314-1317.

7. Azadzoi KM, Babayan RK, Kozlowski R, Siroky MB. Chronic ischemia increases prostatic smooth muscle contraction in the rabbit. J Urol. 2003; 170: 659-663.

8. Kyprianou N, Benning CM. Suppression of human prostate cancer cell growth by alpha 1-adrenoreceptor antagonists doxazosin and terazosin via induction of apoptosis. Cancer Res. 2000; 60: 4550-4555.

9. Morelli A, Sarchielli E, Comegiol P, et al. Phosphodiesterase type 5 expression in human and rat lower urinary tract tissues and the effect of tadalafil on prostate gland oxygenation in spontaneously hypertensive rats. J Sex Med. 2011; 8: 2746-2760.

10. Andesson KE, de Groat WC, McVary KT, et al. Tadalafil for the treatment of lower urinary tract symptoms secondary to benign prostatic hyperplasia: Pathophysiology and mechanism of action. Neurourol Urodyn. 2011; 30: 292-301.

11. Lekas E, Bergh A, Damber JE. Effects of finasteride and bicalutamide on prostatic blood flow in the rat. BJU Int. 2000; 85: 962-965.

12. Mine $\mathrm{S}$, Yamamoto $\mathrm{T}$, Mizuno $\mathrm{H}$, et al. Effect of tamsulosin on bladder microcirculation in rat model of bladder outlet obstruction using pencil lens charge-coupled device microscopy system. Urology. 2013; 81: 155-159.

13. Moher D, Liberati A, Tetzlaff J, Altman DG; PRISMA Group. Preferred reporting items for systematic reviews and meta-analyses: the PRISMA statement. J Clin Epidemiol. 2009; 62: 1006-1012.

14. Higgins JPT, Sterne JAC, Savovic J, et al. A revised tool for assessing risk of bias in randomized trials. In: Chandler J, McKenzie J, Boutron I, Welch V, editors. Cochrane Methods. ISA: Cochrane Database of Systematic Reviews; 2016.

15. Viswanathan M, Ansari MT, Berkman ND, et al. Assessing the risk of bias on individual studies in systematic reviews of health care interventions, In: Methods guide for effectiveness and comparative effectiveness reviews. AHRQ methods for effective health care. Rockville, MD: Agency for Healthcare Research and Quality (US) 2008. Available at: http://www.ncbi.nlm.nih.gov/books/ NBK91433/ accessed: June 12, 2020.

16. Berkman ND, Lohr KN, Ansari M, et al. Grading the strength of a body of evidence when assessing health care interventions for the effective health care program of the Agency for Healthcare Research and Quality: an update. AHRQ Publication No. 13(14)- EHC130-EF. Rockville, MD: Agency for Healthcare Research and Quality. November 2013. www. effectivehealthcare.ahrq.gov/ reports/ final.cfm

17. Popay J, Roberts $H$, Sowden A, Petticrew M, Arai L, Rodgers M, Britten N. Guidance on the conduct of narrative synthesis in systematic reviews. ESRC Research Methods Programme; 2006.

18. Lepor H, Williford WO, Barry MJ, et al. The efficacy of terazosin, finasteride, or both in benign prostatic hyperplasia. N Eng J Med. 1996; 335: 533-539.

19. McConnell JD, Roehrborn CG, Bautista OM, et al. The long-term effects of doxazoin, finasteride, and combination therapy on the clinical progression of benign prostatic hyperplasia. N Eng J Med. 2003; 349: 2387-2398.

20. Yokoyama O, Yoshida M, Kim SC, Wang CJ, Imaoka T, Morisaki Y. Tadalafil once daily for lower urinary tract symptoms suggestive of benign prostatic hyperplasia: A randomize placebo- and tamsulosincontrolled 12-week study in Asian men. Int J Urol. 2013; 20: 193-201.

21. Roehrborn CG for the ALTESS Study Group. Alfuzosin $10 \mathrm{mg}$ once daily prevents overall clinical progression of benign prostatic hyperplasia but not acute urinary retention: results of a 2-year placebocontrolled study. BJU Int. 2006; 97: 734-741.

22. Roehrborn CG. Three months' treatment with the $\alpha 1$-blocker alfuzosin does not affect total of transition zone volume of the prostate. Prostate Cancer Prostatic Dis. 2006; 9: 121-125.

23. Turkeri LN, Ozyurek M, Ersev D, Akdas A. Apoptotic regression of prostatic tissue induced by short-term doxazosin treatment in benign prostatic hyperplasia. Arch Esp de Urol. 2001; 54: 191-196.

24. Debruyne F, Koch G, Boyle P, et al. Comparison of a phytotherapeutic 
agent (Permixon) with an $\alpha$-blocker (tamsulosin) in the treatment of benign prostatic hyperplasia: A 1-year randomized international study. Eur Urol. 2002; 41: 497-507.

25. Sengupta G, Hazra A, Kundu A, Ghosh A. Comparison of Murraya koenigii- and Tribulus terrestris-based oral formulation versus tamsulosin in the treatment of benign prostatic hyperplasia in men aged $>50$ years: a double-blind, double-dummy, randomized controlled trial. Clin Ther. 2011; 33: 1943-1952.

26. Latil A, Petrissans MT, Rouquet J, Robert G, de la Taille A. Effects of hexanic extract of Serenoa repens (Permixon ${ }^{\circledR} 160 \mathrm{mg}$ ) on inflammation biomarkers in the treatment of lower urinary tract symptoms related to benign prostatic hyperplasia. Prostate. 2015; 75: 1857-1867.

27. Pande S, Hazra A, Kundu AK. Evaluation of silodosin in comparison to tamsulosin in benign prostatic hyperplasia. Indian J Pharmacol. 2014; 46: 601-607.

28. Karami H, Hassanzadeh-Hadad A, FallahKarkan M. Comparing monotherapy with tadalafil or tamsulosin and their combination therapy in men with benign prostatic hyperplasia: A randomized clinical trial. Urol J. 2016; 13: 2920-2926.

29. Hizli F, Uygur MC. A prospective study of the efficacy of Serenoa repens, tamsulosin, and Serenoa repens plus tamsulosin treatment for patients with benign prostate hyperplasia. Int Urol Nephrol. 2007; 39: 879-886.

30. Odusanya BO, Tijani KH, Jeje EA, Ogunjimi MA, Ojewola RW. Short-term effect of tamsulosin and finasteride monotherapy and their combination on Nigerian men with benign prostatic hyperplasia. Nig J Surg. 2017; 23: 5-10.

31. Morgia G, Russo GI, Voce S, et al. Serenoa repens, Lycopene and Selenium versus tamsulosin for the treatment of LUTS/ $\mathrm{BPH}$. An Italian multicentre double-blinded randomized study between single or combination therapy (PROCOMB Trial). Prostate 2014; 74: 1471-1480.

32. Roerhborn CG, Siami P, Barkin J, et al. The effects of combination therapy with dutasteride and tamsulosin on clinical outcomes in men with symptomatic benign prostatic hyperplasia: 4-year results from the CombAT Study. Eur Urol. 2010; 57: 123-131.
33. Debruyne FMJ, Jardin A, Colloi D, et al. Sustained-release alfuzosin, finasteride and the combination of both in the treatment of benign prostatic hyperplasia. Eur Urol. 1998; 34: 169-175.

34. Sakalis V, Sfiggas V, Vouros I, Salpiggidis G, Papathanasiou A, Apostolidis A.

Combination of solifenacin with tamsulosin reduces prostate volume and vascularity as opposed to tamsulosin monotherapy in patients with benign prostate enlargement and overactive bladder symptoms: Results from a randomized pilot study. Int J Urol. 2018; 25: 737-745

35. Andersen JT, Ekman P, Wokf H, et al. Can finasteride reverse the progress of benign prostatic hyperplasia: A two-year placebo controlled study. Urology. 1995; 46: 631-637.

36. Nickel JC, Fradet Y, Boake R, et al. Efficacy and safety of finasteride therapy for benign prostatic hyperplasia: Result of a 2-year randomized controlled trial (the PROSPECT Study). Can Med Assoc J. 1996; 155: 1251-1259.

37. McConnell JD, Bruskewitz R, Walsh P, et al. The effect of finasteride on the risk on the risk of acute urinary retention and the need for surgical treatment among men with benign prostatic hyperplasia. N Eng J Med. 1998; 338: 557-563.

38. Marberger MJ, on behalf of the PROWESS Study Group. Long-term effects of finasteride in patients with benign prostatic hyperplasia: A double-blind, placebo-controlled, multicentre study. Urology. 1998; 51: 677-686.

39. Kirby RS, Bryan J, Eardley I, Christmas TJ, Liu S, Holmes SAV, Vale JA, Shanmuganathan K, Webb JA. Finasteride in the treatment on benign prostatic hyperplasia. A urodynamic evaluation. Br J Urol. 1992; 70: 65-72.

40. Stoner $E$, on behalf of The finasteride Study group. The clinical effects of a $5 \alpha$-reductase inhibitor, finasteride, on benign prostatic hyperplasia. J Urol. 1992; 147: 1298-1302.

41. Tammela TLJ, Kontturi MJ. Long-term effects of finasteride on invasive urodynamics and symptoms in the treatment of patients with bladder outflow obstruction due to benign prostatic hyperplasia. J Urol. 1995; 154: 1466-1469.

42. Pannek J, Marks LS, Pearson JD, et al. Influence of finasteride on free and total serum prostate specific antigen levels in men with benign prostatic hyperplasia. J Urol. 1998; 159: 449-453.

43. Marks LS, Partin AW, Gormley GL, et al. Prostate tissue composition and response to finasteride in men with symptomatic benign prostatic hyperplasia. J Urol. 1997; 157: 2171-2178.

44. Gormley GJ, Stoner E, Bruskewitz RC, et al. the effect of finasteride in men with benign prostatic hyperplasia. N Eng J Med. 1992; 327: 1185-1191.

45. Roerhborn CG, Boyle P, Nickel JC, Hoefner K, Andriole G. Efficacy and safety of a dual inhibitor of 5-alpha-reductase types 1 and 1 (Dutasteride) in men with benign prostatic hyperplasia. Urology. 2002; 60: 434-441.

46. Na Y, Ye Z, Zhang S, on behalf of the Chinese Dutasteride Phase III trial (ARIA108898) Study group. Efficacy and safety of dutasteride in Chinese men with benign prostatic hyperplasia. Clin Drug Investig. 2012; 32: 29-39.

47. Tsukamoto T, Endo Y, Narita M. Efficacy and safety of dutasteride in Japanese men with benign prostatic hyperplasia. Int J Urol. 2009; 16: 747-750.

48. Andriole G, Bostwick DG, Brawley OW, et al. Effect of dustasteride on the risk of prostate cancer. N Eng J Med. 2010; 362: 1192-1202.

49. Nickel JC, Gilling P, Tammela TL, Morrill B, Wilson TH, Rittmaster RS. Comparison of dutasteride and finasteride for treating benign protatic hyperplasia: the Enlarged Prostate International Comparator Study (EPICS). BJU Int. 2011; 108: 388-394.

50. Carraro JC, Raynaud JP, Koch G, et al. Comparison of phytotherapy (Permixon ${ }^{\circledR}$ ) with finasteride in the treatment of benign prostate hyperplasia: A randomized international study of 1098 patients. Prostate. 1996; 29: 231-240.

51. Kuo HC, Comparative study of therapeutic effect of dibenyline, finasteride, and combination drugs for symptomatic benign prostatic hyperplasia. Urol Int. 1998; 60: 85-91.

52. Jeong YB, Kwon KS, Kim SD, Kim HJ. Effect of discontinuation of $5 \alpha$-reductase inhibitors on prostate volume and symptoms in men with $\mathrm{BPH}$ : A prospective study. Urology. 2009; 73: 802-806. 
53. Pinggera GM, Frauscher F, Paduch DA, et al. Effect of tadalafil once daily on prostate blood flow and perfusion in men with lower urinary tract symptoms secondary to benign prostatic hyperplasia: A randomized, double-blind, multicenter, placebo-controlled trial. Urology. 2014; 84: 412-420.

54. Morgia G, Vespasiani G, Pareo RM, et al. Serenoa repens + selenium + lycopene vs tadalafil $5 \mathrm{mg}$ for the treatment of lower urinary tract symptoms secondary to benign prostatic obstruction: a Phase IV, non-inferiority, open-label, clinical study (SPRITE study). BJU Int. 2018; 122: 317-325.

55. Kosilov KV, Kuzina IG, Kuznetsov V, Kosilova EK. Improvement of the symptoms of lower urinary tract and sexual function with tadalafil and solifenacin after the treatment of bening prostatic hyperplasia with dutasteride. Prostate Int. 2020; 8: 78-84.

56. Ozturk MI, Kalkan S, Koca O, Gunes M, Akyuz M, Karaman MI. Efficacy of alfuzosin and sildenafil combination in male patients with lower urinary tract symptoms. Andrologia. 2012; 44: 791-795.

57. Joo KJ, Sung WS, Park SH, Yang WJ, Kim TH. Comparison of $\alpha$-blocker monotherapy and $\alpha$-blocker plus $5 \alpha$-reductase inhibitor combination therapy based on prostate volume for treatment $\mathrm{f}$ benign prostatic hyperplasia. J Int Med Res. 2012; 40: 899-908.

58. Choi JD, Kim JH, Ahn SH. Transitional zone index as a preditor of the efficacy of $\alpha$-blocker and $5 \alpha$-reductase inhibitor combination therapy in Korean patients with benign prostatic hyperplasia. Urol Int. 2016; 96: 406-412.

59. Mohanty NK, Singh UP, Sharma NK, Arora RP, Amtabh V. A comparative study of fixed dose of tamsulosin with finasteride vs tamsulosin with dutasteride in the management of benign prostatic hyperplasia. Indian J Urol. 2006; 22: 130-134.

60. Yamanishi T, Asakura H, Seki N, Tokunaga S. Efficacy and safety of combination therapy with tamsulosin, dutasteride and imidafenacin for the management of overactive bladder symptoms associated with benign prostatic hyperplasia: A multicenter, randomized, open-label, controlled trial (DIrect Study). Int J Urol. 2017; 24: 525-531.
61. Ryu YW, Lim SW, Kim JH, Ahn SH, Choi JD. Comparison of tamsulosin plus serenoa repens with tamsulosin in the treatment of benign prostatic hyperplasia in Korean men: 1-year randomized open label study. Urol Int. 2015; 94: 187-193.

62. Argirovic A, Argirovic D. Does the addition of Serenoa repens to tamsulosin improve its therapeutical efficacy in benign prostatic hyperplasia? Vojnosanit Pregl. 2013; 70: 1091-1096.

63. Beiraghdar F, Einollahi B, Panahi Y, Hadjiakhoondi A, Vazirian M, Salarytabar A, Darvishi B. A two-week, double-blind, placebo controlled trial of viola odorata, Echiumamoneum and Physalis alkekengi mixture in symptomatic benign prostate hyperplasia (BPH) in men. Pharm Biol. 2017; 55: 1800-1805.

64. Berges RR, Windeler J, Trampisch HJ, Senge Th and the $\beta$-sitosterol study group. Randomised, placebo-controlled, doubleblind clinical trial of $\beta$-sitosterol in patients with benign prostatic hyperplasia. Lancet. 1995; 345: 1529-1532.

65. Safarinejad MR. Urtica dioca for treatment of benign prostatic hyperplasia: A prospective, randomized, double-blind, placebo-controlled, crossover study. J Herb Pharmacother. 2005; 5: 1-11.

66. Bent S, Kane C, Shinohara K, Neuhaus J, Hudes ES, Goldberg H, Avins AL. Saw Palmetto for benign prostatic hyperplasia. N Eng J Med. 2006; 354: 557-566.

67. Marks LS, Partin AW, Epstein JI, et al. Effects of a saw palmetto herbal blend in men with symptomatic benign prostatic hyperplasia. J Urol. 2000; 163: 1451-1456.

68. Ye Z, Huang J, Zhou L, et al. Efficacy and safety of Serenoa repens extract among patients with benign prostatic hyperplasia in China: A multicenter, randomized, double-blind, placebo-controlled trial. Urology. 2019; 129: 172-179.

69. Zhang W, Wang X, Liu Y, et al. Effects of dietary flaxseed lignin extract on symptoms of benign prostatic hyperplasia. J Med Food. 2008; 11: 207-214.

70. Shi R, Xie Q, Gang X, et al . Effect of saw palmetto soft gel capsule on lower urinary tract symptoms associated with benign prostatic hyperplasia: a randomized trial in Shanghai, China. J Urol. 2008; 179: 610-615.
71. Guzman R, Fernandez JC, Perdoso M, et al. Efficacy and tolerability of Roystonea regia lipid extract (D-004) and terazosin in men with symptomatic benign prostatic hyperplasia: a 6-month study. Ther Adv Urol. 2019; 11: 1-12.

72. Braeckman J, Bruhwyler J, Vandekerckhove K, Geczy J. Efficacy and safety of the extract of serenoa repens in the treatment of benign prostatic hyperplasia: Therapeutic equivalence between twice and once daily dosage forms. Phytother Res. 1997; 11: 558-563.

73. Allott EH, Csizmadi I, Howard LE, et al. Statin use and longitudinal changes in prostate volume; results from the REduction by DUtasteride of prostate Cancer Events (REDUCE) trial. BJU Int. 2020; 125: 226-233.

74. Mills IW, Crossland A, Patel A, Ramonas H. Atorvastatin treatment for men with lower urinary tract symptoms and benign prostatic enlargement. Eur Urol. 2007. 52: 503-509.

75. Zhang X, Zeng X, Dong L, Zhao X, Qu X. The effects of statins on benign prostatic hyperplasia in elderly patients with metabolic syndrome. World J Urol. 2015; 33: 2071-2077.

76. Safwat AS, Hasanain A, Shahat A, et al. Cholecalciferol for the prophylaxis against recurrent urinary tract infection among patients with benign prostatic hyperplasia: A randomized, comparative study. World J Urol. 2019; 37: 1347-1352.

77. Ghadian A, Rezaei M. Combination therapy with omega-3 fatty acids plus tamsulosin and finasteride in the treatment of men with lower urinary tract symptoms (LUTS) and benign prostatic hyperplasia (BPH). Inflammopharmacology. 2017; 25: 451-458.

78. Di Silverio F, Bosman C, Salvatori M, et al. Combination therapy with rofecoxib and finasteride in the treatment of men with lower urinary tract symptoms (LUTS) and benign prostatic hyperplasia (BPH). Eur Urol. 2005; 47: 72-79.

79. Goodarzi D, Cyrus A, Vishtec HRK, Solhi H, Shirinkar. Effect of celecoxib on benign prostatic hyperplasia: Results of a preliminary study. Urol Sci. 2011; 22: 147-150.

80. Jhang JF, Jiang YH, Kuo HC. Adding cyclooxygenase-2 inhibitor to alpha 
blocker for patients with benign prostate hyperplasia and elevated serum prostate specific antigen could not improve prostate biopsy detection rate but improve lower urinary tract symptoms. Int I Clin Pract. 2013; 67: 1327-1333.

81. Page ST, Hirano L, Gilchriest J, et al. Dutasteride reduces prostate size and prostate specific antigen in older hypogonadal men with benign prostatic hyperplasia undergoing testosterone replacement therapy. J Urol. 2011; 186: 191-197.

82. Kacker R, Harisaran V, Given L, Miner M, Rittmaster R, Morgebtaler A. Dutasteride in men receiving testosterone therapy: a randomised, double-blind study. Andrologia. 2015; 47: 148-152.

83. Chung SD, Chang $\mathrm{HC}$, Chiu B, Liao $\mathrm{CH}$, Kuo HC. The efficacy of additive tolterodine extended release for 1-year in older men with storage symptoms and clinical benign prostatic hyperplasia. Neurourol Urodyn. 2011; 30: 568-571.

84. Okutsu H, Matsumoto S, Hanai T, et al. Effects of tamsulosin on bladder blood flow and bladder function in rats with bladder outlet obstruction. Urology. 2010; 75: 235-240.

85. Pinggera GM, Mitterberger M, Pallwein L, et al. alpha-blockers improve chronic ischaemia of the lower urinary tract in patients with lower urinary tract symptoms. BJU Int. 2008; 101: 319-324.

86. Haggstrom S, Lissbrant IF, Bergh A, Damber JE. Testosterone induces vascular endothelial growth factor synthesis in the ventral prostate in castrated rats. J Urol. 1999; 161: 1620-1625.

87. Rittmaster RS, Norman RW, Thomas LN, Rowden G. Evidence for atrophy and apoptosis in the prostates of men given finasteride. J Clin Endocrinol Metab. 1996; 81: 814-819.

88. Kravchick S, Cytron S, Mamonov A, Peled R, Linov L. Effect of short-term dutasteride therapy on prostate vascularity in patients with benign prostatic hyperplasia: a pilot study. Urology. 2009; 73: 1274-1278.

89. Mitterberger M, Pinggera G, Horninger W, et al. Dutasteride prior to contrast enhances colour Doppler ultrasound prostate biopsy increases prostate cancer detection. Eur Urol. 2008; 53: 112-117.

90. Guh JH, Hwang T, Ko FN, Chueh SC, Lai MK, Teng CM. Antiproliferative effect in human prostatic smooth muscle cells by nitric oxide donor. Mol Pharmacol. 1998; 53: 467-474.

91. Kedia GT, Uckert S, Jonas U,Kuczyk MA, Burchardt M. The nitric oxide pathway in the human prostate: Clinical implications in men with lower urinary tract symptoms. World J Urol. 2008; 26: 603-609.

92. Bertolotto $M$, Trincia E, Zappetti R, Bernich R, Savoca G, Cova MA. Effect of tadalafil on prostate haemodynamics: preliminary evaluation with contrastenhanced US. Radiol Med. 2009; 114: 1106-1114.

93. Rosano GM, Aversa A, Vitale C, Fabbri A, Fini M, Spera G. Chronic treatment with tadalafil improves endothelial function in men with increased cardiovascular risk. Eur Urol. 2005; 47: 214-220.

94. Sahinkanat T, Efe E, Ekerbicer HC, Kucukdurmaz F. Effects of a single dose $20 \mathrm{mg}$ tadalafil on resistive index value of prostate zones. Eur Res J. 2018; 4: 320-325.

95. Morelli G, Pagni R, Mariani C, et al. Results of vardenafil mediated power Doppler ultrasound, contrast enhanced ultrasound and systemaic random biopsies to detect prostate cancer. J Urol. 2011; 185: 2126-2131.

96. Vela-Navarrete R, Alcatraz A, Rodriguez-Antolin A, et al. Efficacy and safety of a hexanic extract of Serenoa repens (Permixon) for the treatment of lower urinary tract symptoms associated with benign prostatic hyperplasia (LUTS/BPH): systematic review and meta-analysis of randomised controlled trials and observational studies. BJU Int. 2018; 122: 1049-1065.

97. Majima T, Matsukawa Y, Funahashi Y, Kato M, Yamamoto T, Gotoh M. The effect of mirabegron on bladder blood flow in a rat model of bladder outlet obstruction. World J Urol. 2019; 38: 2021-2027.

98. Roerhborn CG, Perez IO, Roos EPM, et al. Efficacy and safety of a fixed-dose combination of dutasteride and tamsulosin treatment (Duodart) compared with watchful waiting with initiation of tamsulosin therapy if symptoms do not improve, both provided with lifestyle advice, in the management of treatmentnaive men with moderately symptomatic benign prostatic hyperplasia: 2-year CONDUCT study results. BJU Int. 2015; 116: 450-459.

99. Griwan S, Karthikeyan YR, Kumar M, Singh BJ, Singh SK. Comparative evaluation of naftopidil and tamsulosin in the treatment of patients with lower urinary tract symptoms with bening prostatic hyperplasia. Urol Ann. 2014; 5: 181-186.

100. Persu C, Braschi E, Lavelle J. A review of prospective Clinical Trials for neurogenic bladder: Pharmaceuticals. Cent European J Urol. 2014; 67: 264-269. 